\title{
An Updated PAH Mutational Spectrum of Phenylketonuria in Mexican Patients Attending a Single Center: Biochemical, Clinical-Genotyping Correlations
}

 \\ Ariadna González-del Angel ${ }^{2} \mathbb{D}$, Liliana Fernández-Hernández ${ }^{2} \mathbb{D}$, Sara Guillén-López ${ }^{1}{ }^{\mathbb{D}}$, \\ Lizbeth López-Mejía ${ }^{1}$ (D), Rosa Itzel Carrillo-Nieto ${ }^{1}$ (D) , Leticia Belmont-Martínez ${ }^{1}$ (D) \\ and Cynthia Fernández-Lainez ${ }^{1, *(D)}$
}

check for updates

Citation: Vela-Amieva, M.; Alcántara-Ortigoza, M.A.; Ibarra-González, I.; González-del Angel, A.; Fernández-Hernández, L.; Guillén-López, S.; López-Mejía, L.; Carrillo-Nieto, R.I.; Belmont-Martínez, L.; Fernández-Lainez, C. An Updated PAH Mutational Spectrum of Phenylketonuria in Mexican Patients Attending a Single Center: Biochemical, Clinical-Genotyping Correlations. Genes 2021, 12, 1676. https://doi.org/10.3390/ genes12111676

Academic Editor: David N. Cooper

Received: 27 September 2021

Accepted: 19 October 2021

Published: 23 October 2021

Publisher's Note: MDPI stays neutral with regard to jurisdictional claims in published maps and institutional affiliations.

Copyright: (c) 2021 by the authors. Licensee MDPI, Basel, Switzerland. This article is an open access article distributed under the terms and conditions of the Creative Commons Attribution (CC BY) license (https:// creativecommons.org/licenses/by/ $4.0 /)$.
1 Laboratorio de Errores Innatos del Metabolismo y Tamiz, Subdirección de Medicina Experimental, Instituto Nacional de Pediatría, Secretaría de Salud, Ciudad de Mexico 04530, Mexico; dravelaamieva@yahoo.com (M.V.-A.); sara_guillen@hotmail.com (S.G.-L.); lizbeth712@hotmail.com (L.L.-M.); rosecarrillo.ic@gmail.com (R.I.C.-N.); leticia.belmont@gmail.com (L.B.-M.)

2 Laboratorio de Biología Molecular, Subdirección de Investigación Médica, Instituto Nacional de Pediatría, Secretaría de Salud, Ciudad de Mexico 04530, Mexico; malcantaraortigoza@gmail.com (M.A.A.-O.); ariadnagonzalezdelangel@gmail.com (A.G.-d.A.); dralilianafernandez@gmail.com (L.F.-H.)

3 Unidad de Genética de la Nutrición, Instituto de Investigaciones Biomédicas, UNAM/Instituto Nacional de Pediatría, Ciudad de Mexico 04530, Mexico; icig@unam.mx

* Correspondence: lainezcynthia@hotmail.com; Tel.: +52-1-55-10840900 (ext. 1332)

+ These authors contributed equally.

Abstract: Establishing the genotypes of patients with hyperphenylalaninemia (HPA)/phenylketonuria (PKU, MIM\#261600) has been considered a cornerstone for rational medical management. However, knowledge of the phenylalanine hydroxylase gene $(P A H)$ mutational spectrum in Latin American populations is still limited. Herein, we aim to update the mutational $P A H$ spectrum in the largest cohort of HPA/PKU Mexican patients $(N=124)$ reported to date. The biallelic PAH genotype was investigated by Sanger automated sequencing, and genotypes were correlated with documented biochemical phenotypes and theoretical tetrahydrobiopterin $\left(\mathrm{BH}_{4}\right)$ responsiveness. Patients were biochemically classified as having classic PKU (50\%, 62/124), mild PKU (20.2\%, 25/124) and mild HPA $(29.8 \%, 37 / 124)$. Furthermore, $78.2 \%$ of the included patients $(97 / 124)$ were identified by newborn screening. A total of 60 different pathogenic variants were identified, including three novel ones (c. 23del, c. 625_626insC and c. 1315 + 5_1315 + 6insGTGTAACAG), the main categories being missense changes $(58 \%, 35 / 60)$ and those affecting the catalytic domain $(56.6 \%, 34 / 60)$, and c. $60+5 G>T$ was the most frequent variant $(14.5 \%, 36 / 248)$ mainly restricted $(69.2 \%)$ to patients from the central and western parts of Mexico. These 60 types of variants constituted 100 different biallelic PAH genotypes, with the predominance of compound-heterozygous ones (96/124, 77\%). The expected $\mathrm{BH}_{4}$ responsiveness based on the $P A H$ genotype was estimated in $52 \%$ of patients $(65 / 124)$, mainly due to the p. (Val388Met) (rs62516101) allele. Instead, our study identified 27 null variants with an allelic phenotype value of zero, with a predominance of c. $60+5 \mathrm{G}>\mathrm{T}$, which predicts the absence of $\mathrm{BH}_{4}$ responsiveness. An identical genotype reported in BIOPKUdb was found in 92/124 (74\%) of our patients, leading to a genotype-phenotype concordance in 80/92 (86.9\%) of them. The high number of variants found confirms the heterogeneous and complex mutational landscape of $\mathrm{HPA} / \mathrm{PKU}$ in Mexico.

Keywords: phenylketonuria; rare diseases; tetrahydrobiopterin; phenylalanine; newborn screening; Latin America; PAH molecular spectrum 


\section{Introduction}

Phenylketonuria (PKU; MIM \#261600) is an autosomal recessive disorder caused by the deficiency of the enzyme phenylalanine hydroxylase (PAH; E.C.1.14.16.1), which is encoded by the phenylalanine hydroxylase gene (PAH, MIM*612349; 12q23.2) [1]. To date, more than 1200 pathogenic variants have been described in the international database of patients and genotypes causing hyperphenylalaninemia (HPA)/PKU (BIOPKUdb, www.biopku.com, accessed on 26 August 2021), including those genotypes responsive to tetrahydrobiopterin $\left(\mathrm{BH}_{4}\right)$. PKU prevalence varies worldwide, with an average of 1:10,000 newborns (NB); this is more frequent in Italy (1:2700 NB) and Ireland (1:4500 NB), but it is extremely rare in Thailand (1:212,535 NB) and Japan (1:120,000 NB) [2,3]. In Mexico, the HPA/PKU birth prevalence has been estimated to be 1:27,546 [4]. The main biochemical characteristic of PKU is the elevation of phenylalanine (Phe) concentrations in biological fluids, with a concomitant decrease in tyrosine (Tyr), causing brain damage clinically manifested as intellectual disability, seizures, movement disorders, psychiatric and skin problems, and others [2,5].

Recently, an extensive worldwide description of the $P A H$ molecular landscape has been provided, showing the mutational spectra in PKU/HPA patients from different populations [2,3]. Although data from Mexico are included, the reported sample is small (48 patients) and does not contain individuals from all the states that make up the country [6].

According to the recent European and US guidelines for PKU management, the characterization of the responsible $P A H$ genotype must be performed in all patients diagnosed with PKU/HPA, which also should be correlated mainly with the expected biochemical phenotype, dietary Phe tolerance and the $\mathrm{BH}_{4}$ responsiveness $[7,8]$. The aim of this study was to present an update to the mutational spectrum of $P A H$ in the largest cohort to date of clinically described Mexican PKU patients followed at a single center, showing the genotype/phenotype correlation, with emphasis on the severe c. $60+5 \mathrm{G}>\mathrm{T}$ (rs62514895) founder variant, which is considered to be the most common pathogenic allele in our population [6,9]. Furthermore, herein, we reported three novel variants; in addition, in silico modelling analysis was performed to evaluate the recently described p. (His264Arg) variant (BIOPKUdb) in order to predict its possible pathogenic effect.

\section{Materials and Methods}

\subsection{Ethics Statement}

This study was approved by institutional review boards (2020/014), and written informed consent was obtained from all the participants or their parents. After genotype establishment, all the families received genetic counseling.

\subsection{Subjects}

A total of 142 non-related Mexican patients identified with HPA attending the National Institute of Pediatrics were invited to participate. A scheme workflow is shown in Figure 1, and only the 124 patients bearing biallelic $P A H$ genotypes were included. The geographical origin of participants included patients from 30 out of the 32 states in the country. Clinical and demographic data, including the modality of HPA/PKU diagnosis, either by early detection through newborn screening (NBS) or late clinical diagnosis (CD) were registered. As the c. $60+5 \mathrm{G}>\mathrm{T}$ is the most prevalent pathogenic variant in Mexico [6], its clinically and biochemically related phenotype was described, including brain nuclear magnetic resonance imaging (NMRI), in two homozygous and CD patients. The observed biochemical phenotype of patients (67 males and 57 females) was classified following the three categories established by the highest untreated Phe blood concentration as follows: classical


and mild hyperphenylalaninemia (MHP; blood Phe 120-600 $\mu \mathrm{mol} / \mathrm{L}$ ) [3]. 




Figure 1. Workflow scheme for inclusion in the present study of 124 patients bearing $P A H$ biallelic genotypes. The biochemical characteristics of the four identified patients bearing monoallelic PAH genotypes are shown. The 14 patients with normal $\mathrm{PAH}$ genotypes are currently under study for $\mathrm{BH}_{4}$ defects. Abbreviations: HPA: hyperphenylalaninemia; PAH: phenylalanine hydroxylase gene; Phe: phenylalanine; Tyr: tyrosine; mPKU: mild phenylketonuria; MHP: mild hyperphenylalaninemia.

\subsection{Amino Acid Quantification}

The quantification of Phe and Tyr blood concentrations was performed by highperformance liquid chromatography (HPLC) according to a previously described methodology by Qureshi et al. [10] or by tandem mass spectrometry (MS/MS). The determination of blood amino acid profiles by tandem mass spectrometry was performed using a commercial kit from Perkin Elmer (Neobase kit, Wallac Oy, Turku, Finland), according to the manufacturer's instructions.

\subsection{Genotype Analysis}

Genomic DNA samples were obtained from dried blood spots by the saline precipitation method (Gentra Puregene Blood Kit, Gentra Systems, Minneapolis, MN, USA). Polymerase chain reaction (PCR) amplification and direct automated Sanger sequencing were applied to the thirteen PAH exons (NG_008690.2 RefSeqGene, NM_000277.3) in addition to their exon-intron borders. PCR oligonucleotides and sequencing conditions are presented in Supplementary Materials Table S1 and Appendix A. Identified variants were classified according to BIOPKUdb and/or the literature. Novel variants were classified according to the scoring proposed by the American College of Medical Genetics and Genomics and the Association for Molecular Pathology (ACMG/AMP) [11]. Due to limited available information regarding their functional or clinical relevance, these novel and missense changes were subjected to in silico analysis using the PolyPhen (http://genetics.bwh.harvard.edu/ pph2, accessed on 20 December 2020), PROVEAN (http:/ / provean.jcvi.org/index.php, accessed on 20 December 2020), and Pmut (http:// mmb.irbbarcelona.org/PMut, accessed on 20 December 2020) programs.

Novel intronic variants were assessed by the splicing module integrated in Alamut ${ }^{\circledR}$ Visual version 2.15 software (SOPHiA GENETICS, Lausanne, Switzerland), which include SpliceSiteFinder-like, MaxEntScan, GeneSplicer and NNSPLICE in silico evaluations for 
donor and acceptor splice sites, as well as ESEFinder, RESCUE-ESE and EX-SKIP, to predict potentially deleterious effects on Exonic Splicing Enhancer (ESE) binding sites.

\subsection{Protein Modeling and Mutagenesis in Silico}

The crystallographic structure of human PAH with and without ligands (Protein Data Bank (PDB) codes: $1 \mathrm{KW} 0$ and $2 \mathrm{PAH}$, respectively) was used to localize and analyze the potential pathogenic effect of the recently reported $p$. (His264Arg) variant (BIOPKUdb) by mutagenesis in silico. Pymol software, version 2.3.5, was used for protein analyses and figure construction [12]. $1 \mathrm{KW} 0$ crystal was obtained in the presence of $\mathrm{BH}_{4}$ cofactor and the substrate analogue 3-(2-thienyl)-L-alanine (THA) [13].

\subsection{Genotype-Phenotype Correlation with GPV}

Our observed biochemical phenotype (cPKU, mPKU and MHP) was compared with the Genotype Phenotype Value (GPV) calculated from Allelic Phenotype Value (APV) [14] reported in BIOPKUdb. A genotype-phenotype correlation was considered concordant when the obtained GPV corresponded with the reported cut-off.

\subsection{Genotype-Phenotype Correlation with Identical Genotypes}

In the case in which identical genotypes were available in the BIOPKUdb, their associated phenotypes were compared with those found in the patients of the present work. For new variants not reported in BIOPKUdb, the category "not reported" or "still undetermined" was used.

\subsection{Theoretical $\mathrm{BH}_{4}$ Responsiveness and Recommendation to Test}

BIOPKUdb was used to analyze the $\mathrm{BH}_{4}$ responsiveness related to the genotypes found in the present work. To that end, identical genotypes were investigated. In cases in which genotypes or their $\mathrm{BH}_{4}$ responsiveness had not yet been reported in BIOPKUdb, the analysis was performed based on each allele in homozygous state. Since it is well known that genotypes with a GPV equal to zero are non- $\mathrm{BH}_{4}$ responders $[15,16]$, genotypes with these characteristics were excluded from the analysis. If the patient contained any allele that had been classified as a responder or a slow responder in BIOPKUdb, the recommendation was to test for $\mathrm{BH}_{4}$ responsiveness. This same recommendation to test was also made for all the genotypes that had not yet been reported in BIOPKUdb.

\subsection{Statistical Analysis}

Descriptive statistics (average, minimum and maximum) were determined for clinical data of patients. Allelic frequencies were calculated as the number of alleles of each variant per 100/total number of studied alleles. Comparisons of frequencies were performed using a Chi-squared test with MedCalc software version 20.010 (MedCalc Software Ltd., Ostend, Belgium) considering a $p$ value $<0.05$ as significant.

\section{Results}

From the 124 included patients, 97 (78.2\%) were early detected by NBS, and $27(21.8 \%)$ belonged to the $\mathrm{CD}$ group. Based on the Phe blood concentrations, we observed that $62 / 124$ cases were cPKU (50\%), 25/124 were mPKU (20.2\%) and 37/124 were MHP (29.8\%).

\subsection{PAH Variants}

The identified biallelic PAH genotypes included a total of 60 different pathogenic variants, the most frequent being c. $60+5 \mathrm{G}>\mathrm{T}$ among the 124 patients $(36 / 248$ alleles, $14.5 \%$ ). The frequency and type of these 60 different pathogenic variants are shown in Table 1. Six of them had a statistically significant difference of frequency compared to that reported in BIOPKUdb (Table 1). Most pathogenic changes accounted for missense changes (58\%), affected the catalytic domain (56.6\%) (Figure 2) and involved exon 7 (13, $21.7 \%$, Figure 3 ). 




(a)

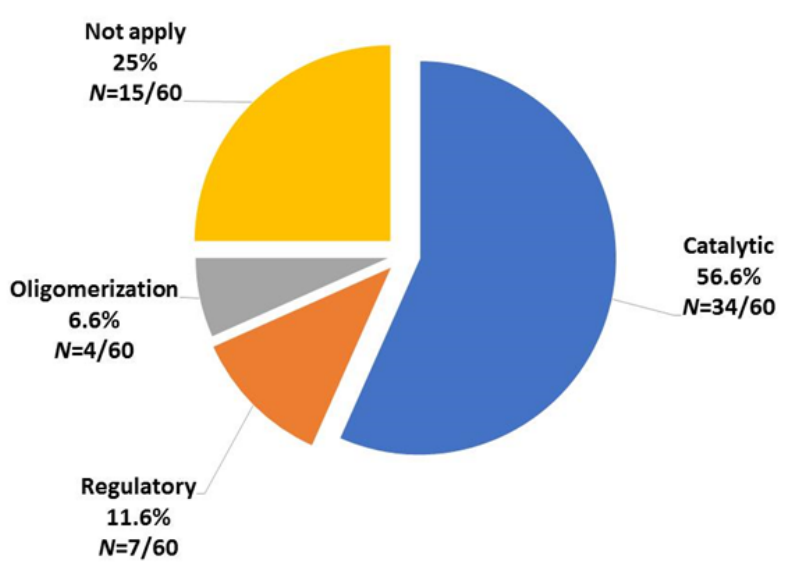

(b)

Figure 2. Main categories of the 60 types of different pathogenic variants identified among the 124 unrelated Mexican patients bearing a diagnostic biallelic PAH genotype. (a) Classification by predicted pathogenic effect of the variant. (b) Classification by affected domain. Frameshift, start-loss, splicing defects and non-sense were considered as null alleles. $N=$ number of alleles/total of alleles (60).

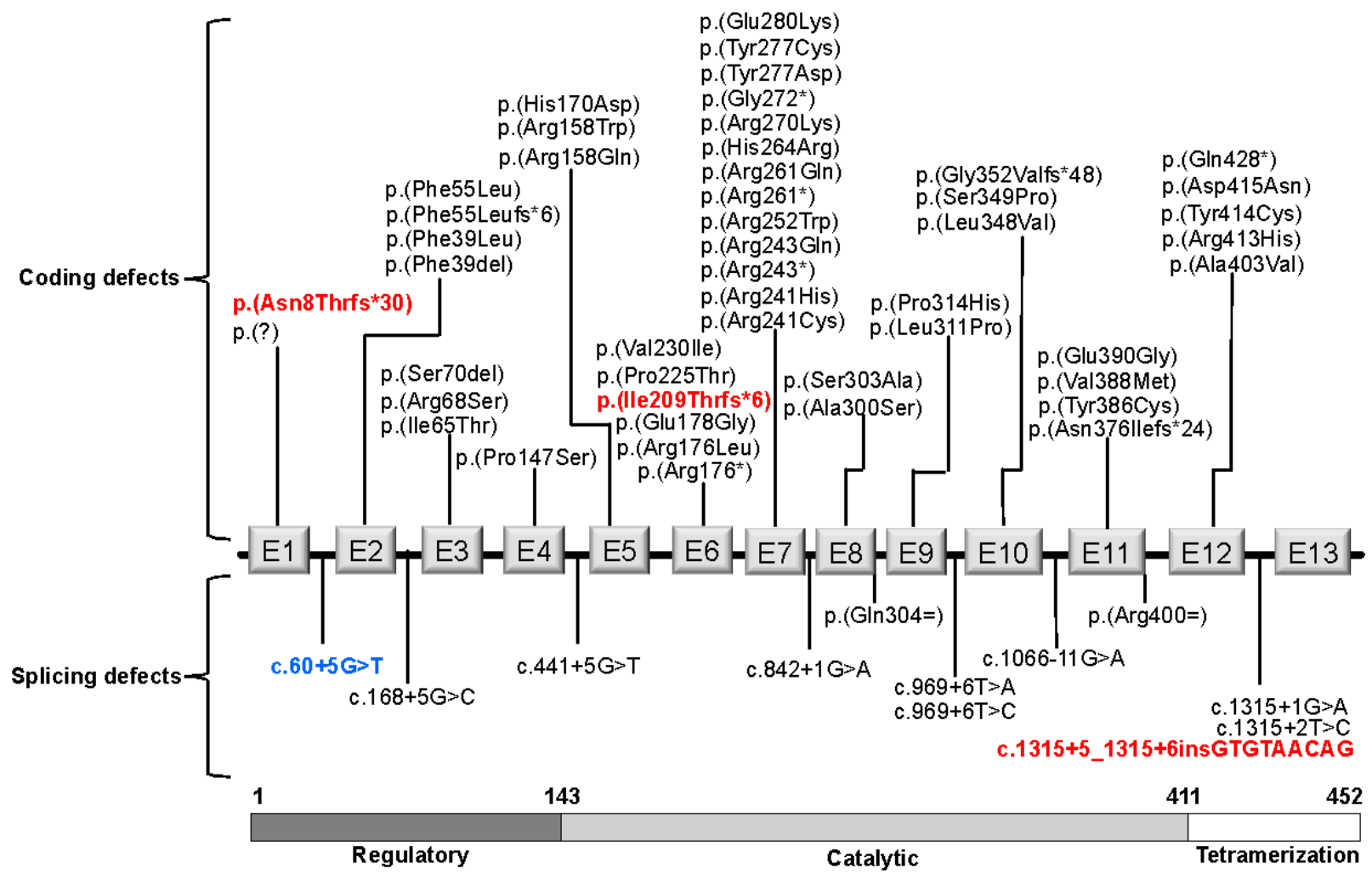

Figure 3. $P A H$ gene diagram showing the localization of the 60 types of variants found in the present study. Novel variants are in red bold type. The most common pathogenic c. $60+5 \mathrm{G}>\mathrm{T}$ splicing variant $(14.5 \%$ of the $P A H$ alleles) is highlighted in blue bold type. The apparently synonymous variants, p. (Gln304=) and p. (Arg400=), disrupt an exon splicing enhancer element, leading to a splicing defect (BIOPKUdb). E1-E13 diagrammatic representations of exons 1 to 13 of PAH. 
Table 1. Frequency and type of $P A H$ variants found in the studied population (248 alleles) and their comparison and categorization accordingly to information available in BIOPKUdb.

\begin{tabular}{|c|c|c|c|c|c|c|c|}
\hline \multirow[b]{2}{*}{$\begin{array}{c}\text { Classification } \\
\text { According APV }{ }^{1}\end{array}$} & \multirow[b]{2}{*}{$P A H$ Variant } & \multirow[b]{2}{*}{ Protein Change } & \multirow[b]{2}{*}{$\begin{array}{l}\% \text { (Present } \\
\text { Work) }\end{array}$} & \multicolumn{4}{|c|}{ BIOPKUdb } \\
\hline & & & & $\%$ & $\mathrm{APV}^{2}$ & EA (\%) & Protein Domain \\
\hline \multirow{42}{*}{$\begin{array}{c}\mathrm{cPKU} \\
(N=42)\end{array}$} & c. $60+5 G>T \mathbf{\square}$ & p. (?) & $14.516 \bullet$ & 0.320 & 0.0 & NR & NA \\
\hline & c. $1162 \mathrm{G}>\mathrm{A}$ & p. (Val388Met) & $11.290 \bullet$ & 1.800 & 1.9 & 28 & Catalytic \\
\hline & c. $441+5 G>T \mathbf{\square}$ & p. (?) & 5.645 & 0.980 & 0.0 & NR & NA \\
\hline & c. $1066-11 G>A \mathbf{\square}$ & p. (Gln355_Tyr356insGlyLeuGln) & 5.645 & 6.800 & 0.0 & 5 & Catalytic \\
\hline & c. $1045 \mathrm{~T}>\mathrm{C} \mathbf{\square}$ & p. (Ser349Pro) & 4.435 & 1.000 & 0.0 & 1 & Catalytic \\
\hline & c. $782 \mathrm{G}>\mathrm{A}$ & p. (Arg261Gln) & 2.823 & 5.500 & 1.6 & 44 & Catalytic \\
\hline & c. $194 \mathrm{~T}>\mathrm{C}$ & p. (Ile65Thr) & 2.419 & 4.000 & 1.0 & 33 & Regulatory \\
\hline & c. $809 \mathrm{G}>\mathrm{A} \boldsymbol{\square}$ & p. (Arg270Lys) & 2.419 & 0.220 & 0.0 & 11 & Catalytic \\
\hline & c. $1 \mathrm{~A}>\mathrm{T} \mathbf{\square}$ & p. (?) & $2.016 \bullet$ & 0.026 & 0.0 & NR & NA \\
\hline & c. $1315+1 G>A \mathbf{\square}$ & p. (?) & 2.016 & 4.400 & 0.0 & NR & NA \\
\hline & c. $728 \mathrm{G}>\mathrm{A} \mathbf{\square}$ & p. (Arg243Gln) & 1.613 & 2.700 & 0.0 & 14 & Catalytic \\
\hline & c. $838 \mathrm{G}>\mathrm{A} \mathbf{\square}$ & p. (Glu280Lys) & 1.613 & 1.300 & 0.0 & 11 & Catalytic \\
\hline & c. $842+1 G>A$ & p. (?) & 1.613 & 0.440 & 0.0 & NR & NA \\
\hline & c. 1055 del $\mathbf{\square}$ & p. (Gly352Valfs * 48) & 1.613 & 0.590 & 0.0 & NR & Catalytic \\
\hline & c. $208 \_210$ delTCT & p. (Ser70del) & 1.210 & 0.026 & 0.7 & NR & Regulatory \\
\hline & c. $673 \bar{C}>\mathrm{A} \square$ & p. (Pro225Thr) & 1.210 & 0.170 & 0.0 & NR & Catalytic \\
\hline & c. $754 \mathrm{C}>\mathrm{T} \mathbf{\square}$ & p. (Arg252Trp) & 1.210 & 1.400 & 0.0 & 15 & Catalytic \\
\hline & c. $781 C>\mathrm{T} \overline{\mathbf{a}}$ & p. $($ Arg261*) & 1.210 & 0.770 & 0.0 & NR & Catalytic \\
\hline & c. $830 \mathrm{~A}>\mathrm{G} \mathbf{\square}$ & p. (Tyr277Cys) & 1.210 & 0.016 & 0.0 & NR & Catalytic \\
\hline & c. $969+6 \mathrm{~T}>\mathrm{A}$ & p. (?) & 1.210 & 0.005 & NR & NR & NA \\
\hline & c. $1042 C>G$ & p. (Leu348Val) & 1.210 & 0.940 & 1.5 & 25 & Catalytic \\
\hline & c. $117 \mathrm{C}>\mathrm{G}$ & p. (Phe39Leu) & 0.806 & 0.420 & 0.2 & 49 & Regulatory \\
\hline & c. $165 \mathrm{del}$ & p. (Phe55Leufs *6) & 0.806 & 0.910 & 0.0 & NR & Regulatory \\
\hline & c. $439 \mathrm{C}>\mathrm{T} \mathbf{\square}$ & p. (Pro147Ser) & 0.806 & 0.042 & 0.0 & NR & Catalytic \\
\hline & c. $625 \_626 \mathrm{insC}$ & p. (Ile209Thrfs *6) & 0.806 & NR & NR & NR & NA \\
\hline & c. $727 \mathrm{C}>\mathrm{T} \boldsymbol{\square}$ & p. $\left(\operatorname{Arg} 243^{*}\right)$ & 0.806 & 1.200 & 0.0 & NR & Catalytic \\
\hline & c. $791 \mathrm{~A}>\mathrm{G}$ & p. (His264Arg) & 0.806 & NR & NR & NR & Catalytic \\
\hline & c. $1157 \mathrm{~A}>\mathrm{G} \mathbf{\square}$ & p. (Tyr386Cys) & 0.806 & 0.074 & 0.0 & NR & Catalytic \\
\hline & c. $23 \mathrm{del}$ & p. (Asn8Thrfs *30) & 0.403 & NR & NR & NR & NA \\
\hline & c. 116_118delTCT & p. (Phe39del) & 0.403 & 0.130 & 0.9 & NR & Regulatory \\
\hline & c. $168+5 G>C \mathbf{\square}$ & p. (?) & 0.403 & 0.600 & 0.0 & NR & NA \\
\hline & c. $472 \mathrm{C}>\mathrm{T}$ & p. (Arg158Trp) & 0.403 & 0.110 & 0.0 & 2 & Catalytic \\
\hline & c. $473 \mathrm{G}>\mathrm{A} \mathbf{\square}$ & p. (Arg158Gln) & 0.403 & 2.900 & 0.0 & 10 & Catalytic \\
\hline & c. $526 \mathrm{C}>\mathrm{T} \mathbf{\square}$ & p. $\left(\operatorname{Arg} 176^{*}\right)$ & 0.403 & 0.410 & 0.0 & NR & Catalytic \\
\hline & c. $814 \mathrm{G}>\mathrm{T} \overline{\mathbf{a}}$ & p. $($ Gly $272 *)$ & 0.403 & 0.790 & 0.0 & NR & Catalytic \\
\hline & c. $829 \mathrm{~T}>\mathrm{G}$ & p. (Tyr277Asp) & 0.403 & 0.240 & 0.4 & NR & Catalytic \\
\hline & c. $932 \mathrm{~T}>\mathrm{C} \mathbf{\square}$ & p. (Leu311Pro) & 0.403 & 0.170 & 0.0 & 10 & Catalytic \\
\hline & c. $1127 \mathrm{del}$ & p. $($ Asn376Ilefs * 24) & 0.403 & 0.005 & 1.0 & NR & Catalytic \\
\hline & c. $1198 \mathrm{~A}>\mathrm{C}$ & p. $(\operatorname{Arg} 400=)$ & 0.403 & 0.005 & NR & NR & NÁ \\
\hline & c. $1282 \mathrm{C}>\mathrm{T} \mathbf{\square}$ & p. $(\mathrm{G} \ln 428 *)$ & $0.403 \star$ & 0.000 & 0 & NR & Oligomerization \\
\hline & c. $1315+2 \mathrm{~T}>\mathrm{C}$ & p. (?) & 0.403 & 0.026 & 0.0 & NR & NA \\
\hline & $\begin{array}{l}\text { c. } 1315+5 \_1315+ \\
\text { 6insGTGTAACAG }\end{array}$ & p. (?) & 0.403 & NR & NR & NR & NA \\
\hline \multirow{6}{*}{$\begin{array}{l}\mathrm{mPKU} \\
(N=6)\end{array}$} & c. $204 \mathrm{~A}>\mathrm{T}$ & p. (Arg68Ser) & $1.210^{\star}$ & 0.600 & 5.4 & 25 & Regulatory \\
\hline & c. $721 \mathrm{C}>\mathrm{T}$ & p. (Arg241Cys) & 0.403 & 1.100 & 5.5 & 57 & Catalytic \\
\hline & c. $722 \mathrm{G}>\mathrm{A}$ & p. (Arg241His) & 2.419 & 0.460 & 5.2 & 23 & Catalytic \\
\hline & c. $912 \mathrm{G}>\mathrm{A}$ & p. $(G \ln 304=)$ & 0.806 & 0.095 & 3.3 & NR & NA \\
\hline & c. $969+6 \mathrm{~T}>\mathrm{C}$ & p. (?) & 0.403 & 0.005 & NR & NR & NA \\
\hline & c. $1241 \mathrm{~A}>\mathrm{G}$ & p. (Tyr414Cys) & 0.806 & 3.100 & 5.1 & 57 & Oligomerization \\
\hline \multirow{12}{*}{$\begin{array}{c}\text { MHP } \\
(N=12)\end{array}$} & c. $165 \mathrm{~T}>\mathrm{G}$ & p. (Phe55Leu) & 0.403 & 0.130 & 8.2 & NR & Regulatory \\
\hline & c. $508 \mathrm{C}>\mathrm{G}$ & p. (His170Asp) & 1.613 & 0.053 & 10.0 & 43 & Catalytic \\
\hline & c. $527 \mathrm{G}>\mathrm{T}$ & p. (Arg176Leu) & 0.403 & 0.150 & 9.8 & 42 & Catalytic \\
\hline & c. $533 \mathrm{~A}>\mathrm{G}$ & p. (Glu178Gly) & 2.419 & 0.300 & 7.6 & 39 & Catalytic \\
\hline & c. $688 \mathrm{G}>\mathrm{A}$ & p. (Val230Ile) & 0.403 & 0.270 & 10.0 & 63 & Catalytic \\
\hline & c. $898 \mathrm{G}>\mathrm{T}$ & p. (Ala300Ser) & 0.403 & 1.500 & 9.7 & 65 & Catalytic \\
\hline & c. $907 \mathrm{~T}>\mathrm{G}$ & p. (Ser303Ala) & 0.403 & 0.047 & 9.3 & NR & Catalytic \\
\hline & c. $941 \mathrm{C}>\mathrm{A}$ & p. (Pro314His) & 0.403 & 0.037 & 10.0 & NR & Catalytic \\
\hline & c. $1169 \mathrm{~A}>\mathrm{G}$ & p. (Glu390Gly) & 2.419 & 1.300 & 6.9 & 62 & Catalytic \\
\hline & c. $1243 \mathrm{G}>\mathrm{A}$ & p. (Asp415Asn) & 3.226 & 0.440 & 10.0 & 72 & Oligomerization \\
\hline & c. $1208 \mathrm{C}>\mathrm{T}$ & p. (Ala403Val) & 2.016 & 2.400 & 9.7 & 66 & Catalytic \\
\hline & c. $1238 \mathrm{G}>\mathrm{A}$ & p. (Arg413His) & 0.403 & NR & 9.0 & NR & Oligomerization \\
\hline
\end{tabular}

${ }^{1}$ Based on the registry of 16,270 PAH alleles listed in BIOPKUdb. ${ }^{2} \mathrm{APV}=$ allelic phenotype value. Null variants have an APV $=0 . \mathrm{Novel}$ variants identified herein are highlighted in red bold type. Abbreviations: cPKU, classic phenylketonuria; EA, enzyme activity as reported in BIOPKUdb; MHP, mild hyperphenylalaninemia; mPKU, mild phenylketonuria; NA, not applicable (splice, non-sense, frameshift, or translation initiation defects); NR, not reported or still undetermined. Statistically significant difference between the frequency of the present work and the one reported in BIOPKUdb. $\mathbf{R}$ Represents non- $\mathrm{BH}_{4}$ responsive alleles, either in homozygous state or in trans with another non-responder allele. 


\subsection{Genotypes}

We found 100 different biallelic genotypes, most of them (96/124, 77\%) in compound heterozygous state (Table 2). Regarding homozygous genotypes, the c. $[60+5 \mathrm{G}<\mathrm{T}]$; $[60+5 \mathrm{G}>\mathrm{T}]$ was the most frequent one (10/28 patients), followed by the c. $[1162 \mathrm{G}>\mathrm{A}]$; [1162G > A] genotype (5/28 patients). From the total of 100 genotypes, $11 \%$ of them included the c. $60+6 \mathrm{G}>\mathrm{T}$ variant, either in homozygous or compound heterozygous state, in which c. [60 + 5G > T]: [1162G > A] (4 cases) and c. [60 + 5G > A]: [441 + 5G > T] ( 3 cases) predominate for this last category.

\subsection{Genotype/Phenotype Concordance with GPV}

Concordance with the GPV was established in 118/124 cases (95.2\%). In 6/124 (4.8\%) cases, concordance could not be established as the allele was reported as undetermined in the BIOPKUdb. From the remaining 118 cases with information available, 85 were concordant $(72 \%)$ and $33 / 118$ cases were discordant $(28 \%)$.

\subsection{Genotype/Phenotype Concordance with Identical Genotypes Reported in BIOPKUdb}

An identical genotype reported in BIOPKUdb was found in 92 out of 124 patients (74\%). From those 92 subjects, 80 (86.9\%) were concordant with the reported phenotype, and in 12 cases, the phenotype was discordant. The disease severity was under- or overestimated in eight and four cases, respectively (Table 2).

\subsection{Clinical Description of Patients Bearing PAH Genotypes Containing c. $60+5 G>T$ Variant}

This pathogenic variant alters the consensus sequence of the $5^{\prime}$ donor splice site of intron 1 of the $P A H$ gene [17], possibly leading to the abolition of its proper recognition by splicing machinery. A total of 26 patients carried the c. $60+6 \mathrm{G}>\mathrm{T}$ variant, either in homozygous (10/26) or in compound heterozygous (16/26) state (Table 3), and 18/26 (69.2\%) of them came from the Mexican Middle West region called El Bajío (Jalisco, Guanajuato, Michoacán and Aguascalientes States). Sixty five percent of these patients $(17 / 26)$ were detected early by NBS, while the remaining patients belonged to the CD group. Brain NMRI imaging of two c. $60+5 \mathrm{G}>$ T-homozygous patients from the CD group demonstrated damage in the basal ganglia, white matter, and cerebral atrophy (Figure 4). The first patient was a one-year-old male who was detected at 9 months old; his Phe diagnostic value was $1654 \mu \mathrm{M}$, he was clinically diagnosed with autism and severe neurodevelopmental delay, and he had poor outcome after nutritional therapy. The other patient was a female who had a diagnostic Phe value of $1830 \mu \mathrm{M}$, at 10 years old, with severe neurodevelopmental delay and behavioral issues, such as auto-aggression and incontrollable tantrums. She was misdiagnosed with autism. After PKU diagnosis, nutritional therapy was started with good compliance and biochemical Phe controls; however, only a few clinical benefits were observed after treatment, such as a decrease in auto-aggression and tantrums. 
Table 2. Genetic spectrum, demographic, clinical, and biochemical characteristics of the studied Mexican HPA/PKU patients ( $N=124)$.

\begin{tabular}{|c|c|c|c|c|c|c|c|c|}
\hline Patient ID & $P A H$ Genotype & Protein Change & Diagnosis & Sex & $\begin{array}{c}\text { Geographical } \\
\text { Origin }\end{array}$ & $\begin{array}{l}\text { Max Historical } \\
\text { Phe }(\mu \mathrm{mol} / \mathrm{L})\end{array}$ & $\begin{array}{c}\text { Observed } \\
\text { Biochemical } \\
\text { Phenotype }\end{array}$ & $\begin{array}{c}\text { Genotypic } \\
\text { Phenotype } \\
\text { Value (GPV) }\end{array}$ \\
\hline 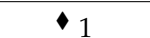 & c. $[60+5 G>T] ;[60+5 G>T]$ & p. [?]; [?] & NBS & $\mathrm{M}$ & Jalisco & 3591 & cPKU & 0 \\
\hline$\cdot 2$ & c. $[60+5 G>T] ;[60+5 G>T]$ & p. [?]; [?] & $\mathrm{CD}$ & $\mathrm{F}$ & CDMX & 2421 & cPKU & 0 \\
\hline 3 & c. $[60+5 G>T]:[60+5 G>T]$ & p. [?]:[?] & $\mathrm{CD}$ & $\mathrm{M}$ & Jalisco & 1896 & cPKU & 0 \\
\hline 4 & c. $[60+5 G>T] ;[60+5 G>T]$ & p. [?]; [?] & $\mathrm{CD}$ & $\mathrm{M}$ & Jalisco & 1654 & $\mathrm{cPKU}$ & 0 \\
\hline 5 & c. $[60+5 G>T] ;[60+5 G>T]$ & p. [?]; [?] & $\mathrm{CD}$ & $\mathrm{M}$ & Jalisco & 1876 & cPKU & 0 \\
\hline 6 & c. $[60+5 \mathrm{G}>\mathrm{T}] ;[60+5 \mathrm{G}>\mathrm{T}]$ & p. [?]; [?] & NBS & $\mathrm{F}$ & Jalisco & 1573 & cPKU & 0 \\
\hline 7 & c. $[60+5 G>T] ;[60+5 G>T]$ & p. [?]; [?] & NBS & $\mathrm{M}$ & Jalisco & 1311 & cPKU & 0 \\
\hline 9 & c. $[60+5 \mathrm{G}>\mathrm{T}] ;[60+5 \mathrm{G}>\mathrm{T}]$ & p. [?]; [?] & NBS & $\mathrm{F}$ & Edo Mex & 1581 & cPKU & 0 \\
\hline 10 & c. $[60+5 \mathrm{G}>\mathrm{T}] ;[60+5 \mathrm{G}>\mathrm{T}]$ & p. [?]; [?] & NBS & $\mathrm{F}$ & Jalisco & 1453 & cPKU & 0 \\
\hline - 11 & c. $[60+5 \mathrm{G}>\mathrm{T}] ;[1162 \mathrm{G}>\mathrm{A}]$ & p. [?]; [Val388Met] & $\mathrm{CD}$ & $\mathrm{F}$ & Puebla & 1900 & cPKU & 1.8 \\
\hline • 12 & c. $[60+5 \mathrm{G}>\mathrm{T}] ;[1162 \mathrm{G}>\mathrm{A}]$ & p. [?]; [Val388Met] & NBS & $\mathrm{M}$ & Jalisco & 3832 & cPKU & 1.8 \\
\hline - 13 & c. $[60+5 \mathrm{G}>\mathrm{T}] ;$ c. $[1162 \mathrm{G}>\mathrm{A}]$ & p. [?]; [Val388Met] & NBS & $\mathrm{M}$ & Aguascalientes & 1876 & $\mathrm{cPKU}$ & 1.8 \\
\hline - 14 & c. $[60+5 \mathrm{G}>\mathrm{T}] ;[1162 \mathrm{G}>\mathrm{A}]$ & p. [?]; [Val388Met] & NBS & $\mathrm{M}$ & Jalisco & 1715 & cPKU & 1.8 \\
\hline 15 & c. $[60+5 \mathrm{G}>\mathrm{T}] ;[441+5 \mathrm{G}>\mathrm{T}]$ & p. [?]; [?] & NBS & $\mathrm{F}$ & Edo Mex & 1682 & cPKU & 0 \\
\hline - 16 & c. $[60+5 \mathrm{G}>\mathrm{T}] ;[441+5 \mathrm{G}>\mathrm{T}]$ & p. [?]; [?] & NBS & $\mathrm{F}$ & Baja California Sur & 1815 & cPKU & 0 \\
\hline - 17 & c. $[60+5 \mathrm{G}>\mathrm{T}] ;[441+5 \mathrm{G}>\mathrm{T}]$ & p. [?]; [?] & $\mathrm{CD}$ & $\mathrm{M}$ & Jalisco & 1346 & $\mathrm{cPKU}$ & 0 \\
\hline - 18 & c. $[60+5 \mathrm{G}>\mathrm{T}] ;[1315+1 \mathrm{G}>\mathrm{A}]$ & p. [?]; [?] & NBS & $\mathrm{F}$ & Guanajuato & 1385 & cPKU & 0 \\
\hline - 21 & c. $[60+5 \mathrm{G}>\mathrm{T}] ;[727 \mathrm{C}>\mathrm{T}]$ & p. [?]; [Arg243*] & $\mathrm{CD}$ & $\mathrm{F}$ & Jalisco & 832 & cPKU & 0 \\
\hline - 22 & c. $[60+5 G>T] ;[1066-11 G>A]$ & p. [?]; [Gln355_Tyr356insGlyLeuGln] & NBS & $\mathrm{F}$ & Jalisco & 1498 & $\mathrm{cPKU}$ & 0 \\
\hline 23 & c. $[60+5 \mathrm{G}>\mathrm{T}] ;[1169 \mathrm{~A}>\mathrm{G}]$ & p. [?]; [Glu390Gly] & NBS & $\mathrm{F}$ & Baja California & 1616 & MHP & 6.9 \\
\hline - 24 & c. $[508 \mathrm{C}>\mathrm{G}] ;[60+5 \mathrm{G}>\mathrm{T}]$ & p. [His170Asp]; [?] & NBS & $\mathrm{M}$ & Jalisco & 1423 & MHP & 10 \\
\hline - 25 & c. $[754 \mathrm{C}>\mathrm{T}] ;[60+5 \mathrm{G}>\mathrm{T}]$ & p. [Arg252Trp]; [?] & $\mathrm{CD}$ & $\mathrm{M}$ & CDMX & 2075 & $\mathrm{cPKU}$ & 0 \\
\hline - 26 & c. $[838 \mathrm{G}>\mathrm{A}] ;[60+5 \mathrm{G}>\mathrm{T}]$ & p. [Glu280Lys]; [?] & $\mathrm{CD}$ & $\mathrm{F}$ & Jalisco & 1949 & $\mathrm{cPKU}$ & 0 \\
\hline 27 & c. $[1162 \mathrm{G}>\mathrm{A}] ;[1162 \mathrm{G}>\mathrm{A}]$ & p. [Val388Met]; [Val388Met] & NBS & $\mathrm{M}$ & Puebla & 1900 & mPKU & 1.8 \\
\hline 28 & c. $[1162 \mathrm{G}>\mathrm{A}] ;[1162 \mathrm{G}>\mathrm{A}]$ & p. [Val388Met]; [Val388Met] & NBS & $\mathrm{F}$ & Puebla & 1516 & mPKU & 1.8 \\
\hline 29 & c. $[1162 \mathrm{G}>\mathrm{A}] ;[1162 \mathrm{G}>\mathrm{A}]$ & p. [Val388Met]; [Val388Met] & $\mathrm{CD}$ & $\mathrm{M}$ & Hidalgo & 1529 & cPKU & 1.8 \\
\hline 30 & c. $[1162 \mathrm{G}>\mathrm{A}] ;[1162 \mathrm{G}>\mathrm{A}]$ & p. [Val388Met]; [Val388Met] & NBS & $\mathrm{M}$ & Queretaro & 2265 & mPKU & 1.8 \\
\hline 31 & c. $[1162 \mathrm{G}>\mathrm{A}] ;[1162 \mathrm{G}>\mathrm{A}]$ & p. [Val388Met]; [Val388Met] & NBS & $\mathrm{F}$ & Queretaro & 2258 & mPKU & 1.8 \\
\hline 32 & c. $[194 \mathrm{~T}>\mathrm{C}] ;[1241 \mathrm{~A}>\mathrm{G}]$ & p. [lle65Thr]; [Tyr414Cys] & NBS & $\mathrm{M}$ & Zacatecas & 790 & mPKU & 5.1 \\
\hline 33 & c. $[194 \mathrm{~T}>\mathrm{C}] ;[1241 \mathrm{~A}>\mathrm{G}]$ & p. [Ile65Thr]; [Tyr414Cys] & NBS & $\mathrm{M}$ & Queretaro & 1070 & mPKU & 5.1 \\
\hline - 34 & c. $[441+5 \mathrm{G}>\mathrm{T}] ;[441+5 \mathrm{G}>\mathrm{T}]$ & p. [?]; [?] & NBS & $\mathrm{F}$ & Tamaulipas & 1640 & mPKU & 0 \\
\hline 35 & c. $[441+5 \mathrm{G}>\mathrm{T}] ;[441+5 \mathrm{G}>\mathrm{T}]$ & p. [?]; [?] & $\mathrm{CD}$ & $\mathrm{M}$ & Coahuila & 1460 & cPKU & 0 \\
\hline - $36^{\Delta}$ & c. $[969+6 \mathrm{~T}>\mathrm{A}] ;[1162 \mathrm{G}>\mathrm{A}]$ & p. [?]; [Val388Met] & NBS & $\mathrm{F}$ & CDMX & 966 & MHP & 1.8 \\
\hline $37^{\Delta}$ & c. $[969+6 \mathrm{~T}>\mathrm{A}] ;[1162 \mathrm{G}>\mathrm{A}]$ & p. [?]; [Val388Met] & NBS & $\mathrm{F}$ & Jalisco & 424 & MHP & 1.8 \\
\hline 38 & c. $[1066-11 \mathrm{G}>\mathrm{A}] ;[1162 \mathrm{G}>\mathrm{A}]$ & $\begin{array}{l}\text { p. [Gln355_Tyr356insGlyLeuGln]; } \\
\text { [Val388Met] }\end{array}$ & $\mathrm{CD}$ & $\mathrm{F}$ & CDMX & 674 & cPKU & 1.8 \\
\hline
\end{tabular}


Table 2. Cont.

\begin{tabular}{|c|c|c|c|c|c|c|c|c|}
\hline Patient ID & PAH Genotype & Protein Change & Diagnosis & Sex & $\begin{array}{c}\text { Geographical } \\
\text { Origin }\end{array}$ & $\begin{array}{l}\text { Max Historical } \\
\text { Phe }(\mu \mathrm{mol} / \mathrm{L})\end{array}$ & $\begin{array}{l}\text { Observed } \\
\text { Biochemical } \\
\text { Phenotype }\end{array}$ & $\begin{array}{c}\text { Genotypic } \\
\text { Phenotype } \\
\text { Value (GPV) }\end{array}$ \\
\hline 39 & c. $[1066-11 \mathrm{G}>\mathrm{A}] ;[1162 \mathrm{G}>\mathrm{A}]$ & $\begin{array}{l}\text { p. [Gln355_Tyr356insGlyLeuGln]; } \\
\text { [Val388Met] }\end{array}$ & NBS & $\mathrm{F}$ & Puebla & 1304 & $\mathrm{mPKU}$ & 1.8 \\
\hline 40 & c. $[1208 \mathrm{C}>\mathrm{T}] ;[1208 \mathrm{C}>\mathrm{T}]$ & p. [Ala403Val]; [Ala403Val] & NBS & M & Queretaro & 1748 & MHP & 9.7 \\
\hline 41 & c. $[1208 \mathrm{C}>\mathrm{T}] ;[1208 \mathrm{C}>\mathrm{T}]$ & p. [Ala403Val]; [Ala403Val] & NBS & $\mathrm{F}$ & CDMX & 1477 & MHP & 9.7 \\
\hline - 42 & c. $[1 \mathrm{~A}>\mathrm{T}] ;$ c. $[1 \mathrm{~A}>\mathrm{T}]$ & p. [?]; [?] & $\mathrm{CD}$ & $\mathrm{M}$ & Veracruz & 1431 & cPKU & 0 \\
\hline - $43^{\Delta}$ & c. $[1 \mathrm{~A}>\mathrm{T}] ;[722 \mathrm{G}>\mathrm{A}]$ & p. [?]; [Arg241His] & NBS & $\mathrm{M}$ & Veracruz & 1225 & MHP & 5.2 \\
\hline 44 & c. $[1 \mathrm{~A}>\mathrm{T}] ;[1042 \mathrm{C}>\mathrm{G}]$ & p. [?]; [Leu348Val] & NBS & $\mathrm{F}$ & CDMX & 1852 & cPKU & 1.5 \\
\hline 45 & c. $[1 \mathrm{~A}>\mathrm{T}] ;[1243 \mathrm{G}>\mathrm{A}]$ & p. [?]; [Asp415Asn] & NBS & $\mathrm{M}$ & Veracruz & 652 & MHP & 10 \\
\hline 46 & c. $[23 \mathrm{del}] ;[1162 \mathrm{G}>\mathrm{A}]$ & p. [Asn8Thrfs * 30]; [Val388Met] & NBS & $\mathrm{F}$ & Chihuahua & 1187 & cPKU & Unknown \\
\hline 47 & c. $[117 \mathrm{C}>\mathrm{G}] ;[441+5 \mathrm{G}>\mathrm{T}]$ & p. [Phe39Leu]; [?] & NBS & M & Chihuahua & 1075 & mPKU & 0.2 \\
\hline 48 & c. $[117 \mathrm{C}>\mathrm{G}] ;[1157 \mathrm{~A}>\mathrm{G}]$ & p. [Phe39Leu]; [Tyr386Cys] & NBS & $\mathrm{F}$ & Durango & 1184 & $\mathrm{mPKU}$ & 0.2 \\
\hline 49 & c. $[165 \mathrm{del}] ;[194 \mathrm{~T}>\mathrm{C}]$ & p. [Phe55Leufs * 6]; [lle65Thr] & NBS & M & Durango & 1066 & cPKU & 1.1 \\
\hline 50 & c. [165T > G]; [208_210del] & p. [Phe55Leu]; [Ser70del] & NBS & M & Chihuahua & 2825 & MHP & 8.2 \\
\hline 51 & c. $[168+5 G>C] ;[1066-11 G>A]$ & p. [?]; [Gln355_Tyr356insGlyLeuGln] & NBS & $\mathrm{M}$ & Chihuahua & 1222 & cPKU & 0 \\
\hline 52 & c. $[194 \mathrm{~T}>\mathrm{C}] ;$ [208_210del] & p. [Ile65Thr]; [Ser70del] & NBS & M & Zacatecas & 1791 & cPKU & 1 \\
\hline $53^{\Delta}$ & c. $[194 \mathrm{~T}>\mathrm{C}] ;[533 \mathrm{~A}>\mathrm{G}]$ & p. [Ile65Thr]; [Glu178Gly] & NBS & $\mathrm{F}$ & Sonora & 1527 & MHP & 7.6 \\
\hline 54 & c. $[204 \mathrm{~A}>\mathrm{T}] ;[527 \mathrm{G}>\mathrm{T}]$ & p. [Arg68Ser]; [Arg176Leu] & NBS & M & CDMX & 799 & MHP & 9.7 \\
\hline • 55 & c. $[204 \mathrm{~A}>\mathrm{T}] ;[782 \mathrm{G}>\mathrm{A}]$ & p. [Arg68Ser]; [Arg261Gln] & NBS & M & Queretaro & 1187 & mPKU & 5.4 \\
\hline 56 & c. $[204 \mathrm{~A}>\mathrm{T}] ;[829 \mathrm{~T}>\mathrm{G}]$ & p. [Arg68Ser]; [Tyr277Asp] & NBS & M & Veracruz & 1255 & MHP & 5.4 \\
\hline - 58 & c. $[439 \bar{C}>\mathrm{T}] ;[1045 \mathrm{~T}>\mathrm{C}]$ & p. [Pro147Ser]; [Ser349Pro] & NBS & M & Jalisco & 2098 & cPKU & 0 \\
\hline - 59 & c. $[439 \mathrm{C}>\mathrm{T}] ;[782 \mathrm{G}>\mathrm{A}]$ & p. [Pro147Ser]; [Arg261Gln] & NBS & $\mathrm{F}$ & Michoacan & 1923 & mPKU & 1.6 \\
\hline - 60 & c. $[441+5 \mathrm{G}>\mathrm{T}] ;[1045 \mathrm{~T}>\mathrm{C}]$ & p. [?]; [Ser349Pro] & NBS & $\mathrm{F}$ & Edo Mex & 1452 & $\mathrm{cPKU}$ & 0 \\
\hline 61 & c. $[441+5 \mathrm{G}>\mathrm{T}] ;[1055 \mathrm{del}]$ & p. [?]; [Gly352Valfs* 48] & NBS & M & CDMX & 1756 & cPKU & 0 \\
\hline • 62 & c. $[441+5 \mathrm{G}>\mathrm{T}] ;[165 \mathrm{del}]$ & p. [?]; [Phe55Leufs*6] & $\mathrm{CD}$ & $\mathrm{F}$ & Chihuahua & 1634 & cPKU & 0 \\
\hline - 63 & c. $[441+5 \mathrm{G}>\mathrm{T}] ;[782 \mathrm{G}>\mathrm{A}]$ & p. [?]; [Arg261Gln] & $\mathrm{CD}$ & M & CDMX & 890 & cPKU & 1.6 \\
\hline 64 & c. $[441+5 \mathrm{G}>\mathrm{T}] ;[791 \mathrm{~A}>\mathrm{G}]$ & p. [?]; [His264Arg] & NBS & $\mathrm{F}$ & Edo Mex & 921 & cPKU & 0 \\
\hline 65 & c. $[473 \mathrm{G}>\mathrm{A}] ;[1045 \mathrm{~T}>\mathrm{C}]$ & p. [Arg158Gln]; [Ser349Pro] & NBS & $\mathrm{M}$ & CDMX & 1656 & cPKU & 0 \\
\hline 66 & c. $[508 \mathrm{C}>\mathrm{G}] ;[1243 \mathrm{G}>\mathrm{A}]$ & p. [His170Asp]; [Asp415Asn] & NBS & M & Jalisco & 1098 & MHP & 10 \\
\hline - 67 & c. $[526 \mathrm{C}>\mathrm{T}] ;[1066-11 \mathrm{G}>\mathrm{A}]$ & $\begin{array}{l}\text { p. [Arg176* * ; } \\
\text { [Gln355_Tyr356insGlyLeuGln] }\end{array}$ & $\mathrm{CD}$ & M & CDMX & 1113 & $\mathrm{cPKU}$ & 0 \\
\hline 68 & c. $[533 \mathrm{~A}>\mathrm{G}] ;[1162 \mathrm{G}>\mathrm{A}]$ & p. [Glu178Gly]; [Val388Met] & NBS & $\mathrm{F}$ & Hidalgo & 502 & MHP & 7.6 \\
\hline 69 & c. $[533 \mathrm{~A}>\mathrm{G}] ;[1169 \mathrm{~A}>\mathrm{G}]$ & p. [Glu178Gly]; [Glu390Gly] & NBS & $\mathrm{M}$ & CDMX & 179 & MHP & 7.6 \\
\hline 70 & c. $[533 \mathrm{~A}>\mathrm{G}] ;[533 \mathrm{~A}>\mathrm{G}]$ & p. [Glu178Gly]; [Glu178Gly] & $\mathrm{CD}$ & M & Queretaro & 1277 & MHP & 7.6 \\
\hline 71 & c. $[533 \mathrm{~A}>\mathrm{G}] ;[809 \mathrm{G}>\mathrm{A}]$ & p. [Glu178Gly]; [Arg270Lys] & NBS & $\mathrm{F}$ & Edo Mex & 1154 & MHP & 7.6 \\
\hline 72 & c. [625_626insC]; [625_626insC] & p. [?]; [?] & NBS & M & Veracruz & 1008 & cPKU & Unknown \\
\hline 73 & c. $[673 \mathrm{C}>\mathrm{A}] ;[830 \mathrm{~A}>\mathrm{G}]$ & p. [Pro225Thr]; [Tyr277Cys] & NBS & M & Quintana Roo & 1022 & mPKU & 0 \\
\hline 74 & c. $[688 \mathrm{G}>\mathrm{A}] ;[754 \mathrm{C}>\mathrm{T}]$ & p. [Val230Ile]; [Arg252Trp] & NBS & $\mathrm{F}$ & Veracruz & 1608 & MHP & 10 \\
\hline
\end{tabular}


Table 2. Cont.

\begin{tabular}{|c|c|c|c|c|c|c|c|c|}
\hline Patient ID & PAH Genotype & Protein Change & Diagnosis & Sex & $\underset{\text { Origin }}{\text { Geographical }}$ & $\begin{array}{l}\text { Max Historical } \\
\text { Phe }(\mu \mathrm{mol} / \mathrm{L})\end{array}$ & $\begin{array}{c}\text { Observed } \\
\text { Biochemical } \\
\text { Phenotype }\end{array}$ & $\begin{array}{c}\text { Genotypic } \\
\text { Phenotype } \\
\text { Value (GPV) }\end{array}$ \\
\hline 75 & c. $[721 \mathrm{C}>\mathrm{T}] ;[1066-11 \mathrm{G}>\mathrm{A}]$ & $\begin{array}{l}\text { p. [Arg241Cys]; } \\
\text { [Gln355 Tyr356insGlyLeuGln] }\end{array}$ & NBS & $\mathrm{M}$ & CDMX & 1747 & MHP & 5.5 \\
\hline 76 & c. $[722 \mathrm{G}>\mathrm{A}] ;[\mathrm{782G}>\mathrm{A}]$ & p. [Arg241His]; [Arg261Gln] & NBS & $\mathrm{F}$ & CDMX & 2396 & MHP & 5.2 \\
\hline $77^{\bullet}$ & c. $[722 \mathrm{G}>\mathrm{A}] ;[842+1 \mathrm{G}>\mathrm{A}]$ & p. [Arg241Cys]; [?] & NBS & $\mathrm{M}$ & Tabasco & 2134 & cPKU & 5.2 \\
\hline 78 & c. $[722 \mathrm{G}>\mathrm{A}] ;[722 \mathrm{G}>\mathrm{A}]$ & p. [Arg241Cys]; [Arg241His] & NBS & M & Puebla & 4627 & MHP & 5.2 \\
\hline 79 & c. $[727 \mathrm{C}>\mathrm{T}] ;[1243 \mathrm{G}>\mathrm{A}]$ & p. [Arg241Cys]; [Asp415Asn] & NBS & $\mathrm{F}$ & CDMX & 948 & MHP & 10 \\
\hline 80 & c. $[728 \mathrm{G}>\mathrm{A}] ;[1243 \mathrm{G}>\mathrm{A}]$ & p. [Arg243Gln]; [Asp415Asn] & NBS & $\mathrm{M}$ & CDMX & 712 & MHP & 10 \\
\hline$\bullet 81^{\Delta}$ & c. $[728 \mathrm{G}>\mathrm{A}] ;[722 \mathrm{G}>\mathrm{A}]$ & p. [Arg243Gln]; [Arg241His] & NBS & $\mathrm{F}$ & CDMX & 506 & MHP & 5.2 \\
\hline 82 & c. $[728 \mathrm{G}>\mathrm{A}] ;[809 \mathrm{G}>\mathrm{A}]$ & p. [Arg243Gln]; [Arg270Lys] & NBS & $\mathrm{M}$ & Chihuahua & 587 & $\mathrm{mPKU}$ & 0 \\
\hline - 83 & c. $[781 \mathrm{C}>\mathrm{T}] ;[1066-11 \mathrm{G}>\mathrm{A}]$ & $\begin{array}{l}\text { p. [Arg261*]; } \\
\text { [Gln355 Tyr356insGlyLeuGln] }\end{array}$ & $\mathrm{CD}$ & $\mathrm{M}$ & Jalisco & 1623 & $\mathrm{cPKU}$ & 0 \\
\hline - 84 & c. $[781 \mathrm{C}>\mathrm{T}] ;[782 \mathrm{G}>\mathrm{A}]$ & p. [Arg261*]; [Arg261Gln] & NBS & $\mathrm{F}$ & CDMX & 177 & cPKU & 1.6 \\
\hline 85 & c. $[781 \mathrm{C}>\mathrm{T}] ;[1243 \mathrm{G}>\mathrm{A}]$ & p. [Arg261 *]; [Asp415Asn] & NBS & $\mathrm{M}$ & CDMX & 470 & MHP & 10 \\
\hline 86 & c. $[782 \mathrm{G}>\mathrm{A}] ;[1045 \mathrm{~T}>\mathrm{C}]$ & p. [Arg261Gln]; [Ser349Pro] & NBS & $\mathrm{M}$ & Nuevo León & 794 & cPKU & 1.6 \\
\hline 87 & c. $[782 \mathrm{G}>\mathrm{A}] ;[1169 \mathrm{~A}>\mathrm{G}]$ & p. [Arg261Gln]; [Glu390Gly] & NBS & $\mathrm{F}$ & Guanajuato & 215 & MHP & 7 \\
\hline 88 & $\begin{array}{l}\text { c. [ }[791 \mathrm{~A}>\mathrm{G}] ; \text { [ }[1315+5 \text { 5_1315 + } \\
\text { 6insGTGTAACAG] }\end{array}$ & p. [His264Arg]; [?] & NBS & $\mathrm{M}$ & Veracruz & 401 & $\mathrm{cPKU}$ & Unknown \\
\hline $89^{\Delta}$ & c. $[809 \mathrm{G}>\mathrm{A}] ;[1162 \mathrm{G}>\mathrm{A}]$ & p. [Arg270Lys]; [Val388Met] & NBS & M & San Luis Potosi & 467 & mPKU & 1.8 \\
\hline 90 & c. $[809 \mathrm{G}>\mathrm{A}] ;[1066-11 \mathrm{G}>\mathrm{A}]$ & $\begin{array}{l}\text { p. [Arg270Lys]; } \\
\text { [Gln355 Tyr356insGlyLeuGln] }\end{array}$ & NBS & $\mathrm{M}$ & Puebla & 282 & $\mathrm{cPKU}$ & 0 \\
\hline 91 & c. $[814 \mathrm{G}>\mathrm{T}] ;[1282 \mathrm{C}>\mathrm{T}]$ & p. [Gly $272 *] ;[G \ln 428 *]$ & NBS & $\mathrm{F}$ & Edo Mex & 566 & mPKU & 0 \\
\hline 92 & c. $[830 \mathrm{~A}>\mathrm{G}] ;[830 \mathrm{~A}>\mathrm{G}]$ & p. [Tyr277Cys]; [Tyr277Cys] & NBS & $\mathrm{M}$ & Yucatan & 454 & MHP & 0 \\
\hline - 93 & c. $[838 \mathrm{G}>\mathrm{A}] ;[[838 \mathrm{G}>\mathrm{A}]$ & p. [Glu280Lys]; [Glu280Lys] & NBS & $\mathrm{F}$ & CDMX & 283 & $\mathrm{mPKU}$ & 0 \\
\hline 94 & c. $[838 \mathrm{G}>\mathrm{A}] ;[1066-11 \mathrm{G}>\mathrm{A}]$ & $\begin{array}{l}\text { p. [Glu280Lys]; } \\
\text { [Gln355 Tyr356insGlyLeuGln] }\end{array}$ & NBS & $\mathrm{F}$ & Colima & 512 & cPKU & 0 \\
\hline$\bullet 95$ & c. $[842+1 G>A] ;[441+5 G>T]$ & p. [?]; [?] & NBS & M & Edo Mex & 276 & cPKU & 0 \\
\hline$\bullet 96^{\bullet}$ & c. $[842+1 G>A] ;[508 C>G]$ & p. [?]; [His170Asp] & $\mathrm{CD}$ & $\mathrm{M}$ & Guanajuato & 239 & mPKU & 10 \\
\hline 97 & c. $[842+1 G>A] ;[932 T>C]$ & p. [?]; [Leu311Pro] & $\mathrm{CD}$ & $\mathrm{F}$ & Sonora & 239 & cPKU & 0 \\
\hline 98 & c. $[898 \mathrm{G}>\mathrm{T}] ;[1162 \mathrm{G}>\mathrm{A}]$ & p. [Ala300Ser]; [Val388Met] & NBS & $\mathrm{F}$ & Queretaro & 289 & MHP & 9.7 \\
\hline - 99 & c. $[907 \mathrm{~T}>\mathrm{G}] ;[673 \mathrm{C}>\mathrm{A}]$ & p. [Ser303Ala]; [Pro225Thr] & NBS & $\mathrm{F}$ & $\widehat{C D M X}$ & 1351 & MHP & 9.3 \\
\hline - 100 & c. $[912 \mathrm{G}>\mathrm{A}] ;[912 \mathrm{G}>\mathrm{A}]$ & p. $[\mathrm{G} \ln 304=] ;[\mathrm{G} \ln 304=]$ & $\mathrm{CD}$ & $\mathrm{F}$ & Sinaloa & 218 & cPKU & 3.3 \\
\hline - 101 & c. $[941 \mathrm{C}>\mathrm{A}] ;[809 \mathrm{G}>\mathrm{A}]$ & p. [Pro314His]; [Arg270Lys] & NBS & $\mathrm{M}$ & Aguascalientes & 245 & $\mathrm{MHP}$ & 10 \\
\hline 102 & c. $[969+6 \mathrm{~T}>\mathrm{A}] ;[1198 \mathrm{~A}>\mathrm{C}]$ & p. [?]; [Arg400=] & NBS & $\mathrm{M}$ & Michoacan & 278 & MHP & Undetermined \\
\hline - 103 & c. $[1042 \mathrm{C}>\mathrm{G}] ;[194 \mathrm{~T}>\mathrm{C}]$ & p. [Leu348Val]; [Ile65Thr] & $\mathrm{CD}$ & $\mathrm{M}$ & Morelos & 180 & mPKU & 1.5 \\
\hline 104 & c. $[1042 C>G] ;[1238 G>A]$ & p. [Leu348Val]; [Arg413His] & NBS & $\mathrm{F}$ & Tabasco & 248 & cPKU & 9 \\
\hline • 105 & c. $[1045 \mathrm{~T}>\mathrm{C}] ;[1066-11 \mathrm{G}>\mathrm{A}]$ & $\begin{array}{l}\text { p. [Ser349Pro]; } \\
\text { [Gln355 Tyr356insGlyLeuGln] }\end{array}$ & NBS & $\mathrm{M}$ & Chihuahua & 242 & $\mathrm{cPKU}$ & 0 \\
\hline - 106 & c. $[1045 \mathrm{~T}>\mathrm{C}] ;[1169 \mathrm{~A}>\mathrm{G}]$ & p. [Ser349Pro]; [Glu390Gly] & NBS & $\mathrm{F}$ & CDMX & 600 & MHP & 7 \\
\hline
\end{tabular}


Table 2. Cont.

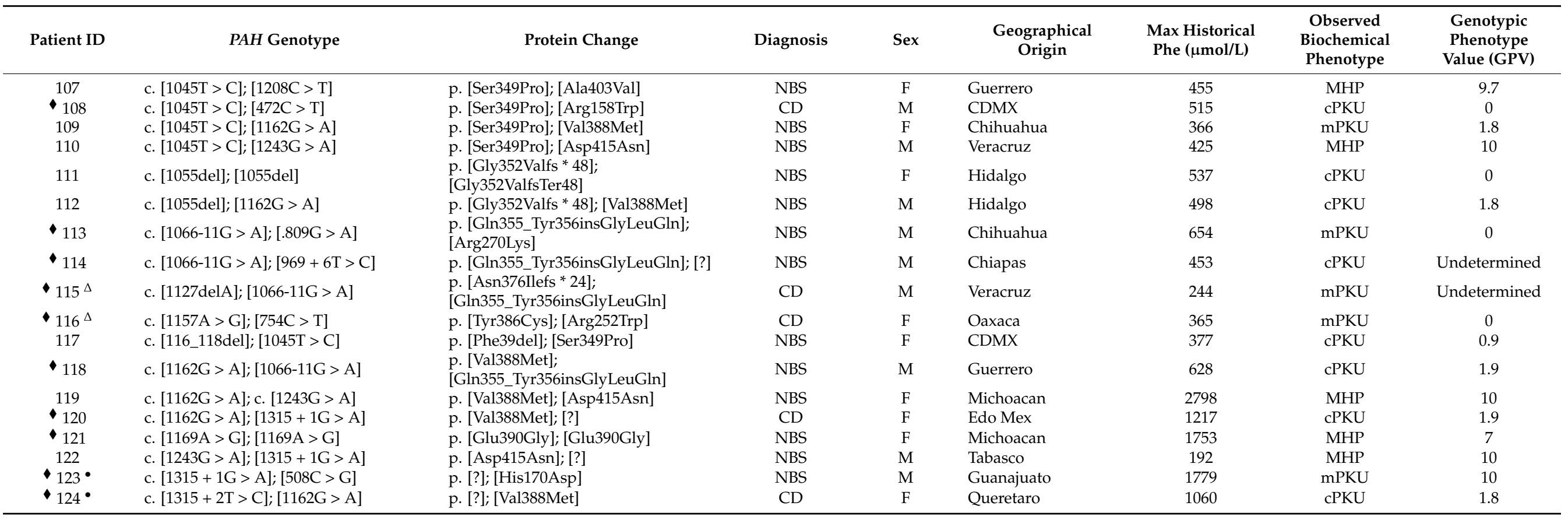

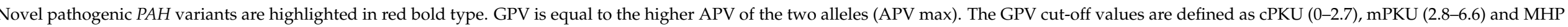

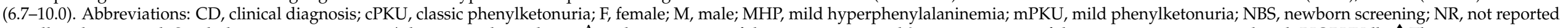

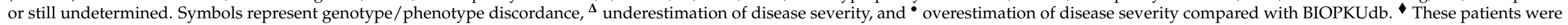
described in our previous report [6]. 
Table 3. Main biochemical and clinical characteristics of the patients harboring the c. $60+5 \mathrm{G}<\mathrm{T}$ variant.

\begin{tabular}{|c|c|c|c|c|c|c|c|}
\hline Patient ID & PAH Genotype & $\begin{array}{c}\text { Observed } \\
\text { Biochemical } \\
\text { Phenotype }\end{array}$ & GPV & Diagnosis & $\begin{array}{c}\text { Max } \\
\text { Historical } \\
\text { Phe }(\mu \mathrm{mol} / \mathrm{L})\end{array}$ & $\begin{array}{c}\text { Tyr } \\
(\mu \mathrm{mol} / \mathrm{L})\end{array}$ & $\begin{array}{c}\text { Phe/Tyr } \\
\text { Ratio }\end{array}$ \\
\hline 1 & c. $[60+5 \mathrm{G}>\mathrm{T}] ;[60+5 \mathrm{G}>\mathrm{T}]$ & cPKU & 0.0 & NBS & 3591 & 70 & 41.26 \\
\hline 2 & c. $[60+5 \mathrm{G}>\mathrm{T}] ;[60+5 \mathrm{G}>\mathrm{T}]$ & cPKU & 0.0 & $\mathrm{CD}$ & 2421 & 64 & 37.83 \\
\hline 3 & c. $[60+5 \mathrm{G}>\mathrm{T}] ;[60+5 \mathrm{G}>\mathrm{T}]$ & cPKU & 0.0 & CD & 1896 & 38 & 49.89 \\
\hline 4 & c. $[60+5 \mathrm{G}>\mathrm{T}] ;[60+5 \mathrm{G}>\mathrm{T}]$ & cPKU & 0.0 & CD & 1654 & 38 & 43.53 \\
\hline 5 & c. $[60+5 \mathrm{G}>\mathrm{T}] ;[60+5 \mathrm{G}>\mathrm{T}]$ & cPKU & 0.0 & CD & 1876 & 42 & 44.67 \\
\hline 6 & c. $[60+5 \mathrm{G}>\mathrm{T}] ;[60+5 \mathrm{G}>\mathrm{T}]$ & cPKU & 0.0 & NBS & 1573 & 61 & 25.79 \\
\hline 7 & c. $[60+5 \mathrm{G}>\mathrm{T}] ;[60+5 \mathrm{G}>\mathrm{T}]$ & cPKU & 0.0 & NBS & 1311 & 33 & 39.73 \\
\hline 8 & c. $[60+5 \mathrm{G}>\mathrm{T}] ;[60+5 \mathrm{G}>\mathrm{T}]$ & cPKU & 0.0 & NBS & 1712 & 39 & 43.90 \\
\hline 9 & c. $[60+5 \mathrm{G}>\mathrm{T}] ;[60+5 \mathrm{G}>\mathrm{T}]$ & cPKU & 0.0 & NBS & 1581 & 35 & 45.17 \\
\hline 10 & c. $[60+5 \mathrm{G}>\mathrm{T}] ;[60+5 \mathrm{G}>\mathrm{T}]$ & cPKU & 0.0 & NBS & 1453 & 109 & 13.33 \\
\hline 11 & c. $[60+5 \mathrm{G}>\mathrm{T}] ;[1162 \mathrm{G}>\mathrm{A}]$ & cPKU & 1.8 & CD & 1923 & 124 & 15.51 \\
\hline 12 & c. $[60+5 \mathrm{G}>\mathrm{T}] ;[1162 \mathrm{G}>\mathrm{A}]$ & cPKU & 1.8 & NBS & 1452 & 26 & 55.85 \\
\hline 13 & c. $[60+5 \mathrm{G}>\mathrm{T}] ;$ c. $[1162 \mathrm{G}>\mathrm{A}]$ & cPKU & 1.8 & NBS & 1756 & 38 & 46.21 \\
\hline 14 & c. $[60+5 \mathrm{G}>\mathrm{T}] ;[1162 \mathrm{G}>\mathrm{A}]$ & cPKU & 1.8 & NBS & 1634 & 106 & 15.42 \\
\hline 15 & c. $[60+5 \mathrm{G}>\mathrm{T}] ;[441+5 \mathrm{G}>\mathrm{T}]$ & cPKU & 0.0 & NBS & 1900 & 36 & 52.78 \\
\hline 16 & c. $[60+5 \mathrm{G}>\mathrm{T}] ;[441+5 \mathrm{G}>\mathrm{T}]$ & cPKU & 0.0 & NBS & 3832 & 78 & 49.13 \\
\hline 17 & c. $[60+5 \mathrm{G}>\mathrm{T}] ;[441+5 \mathrm{G}>\mathrm{T}]$ & cPKU & 0.0 & $\mathrm{CD}$ & 1876 & 58 & 32.34 \\
\hline 18 & c. $[60+5 \mathrm{G}>\mathrm{T}] ;[1315+1 \mathrm{G}>\mathrm{A}]$ & cPKU & 0.0 & NBS & 1715 & 46 & 37.28 \\
\hline 19 & c. $[60+5 \mathrm{G}>\mathrm{T}] ;[1315+1 \mathrm{G}>\mathrm{A}]$ & cPKU & 0.0 & NBS & 1682 & 70 & 24.03 \\
\hline 20 & c. $[60+5 \mathrm{G}>\mathrm{T}] ;[673 \mathrm{C}>\mathrm{A}]$ & cPKU & 0.0 & NBS & 1815 & 69 & 26.30 \\
\hline 21 & c. $[60+5 \mathrm{G}>\mathrm{T}] ;[727 \mathrm{C}>\mathrm{T}]$ & cPKU & 0.0 & $\mathrm{CD}$ & 1346 & 108 & 12.46 \\
\hline 22 & c. $[60+5 \mathrm{G}>\mathrm{T}] ;[1066-11 \mathrm{G}>\mathrm{A}]$ & cPKU & 0.0 & NBS & 1385 & 113 & 12.26 \\
\hline 23 & c. $[60+5 \mathrm{G}>\mathrm{T}] ;[1169 \mathrm{~A}>\mathrm{G}]$ & MHP & 6.9 & NBS & 467 & 103 & 4.53 \\
\hline 24 & c. $[60+5 \mathrm{G}>\mathrm{T}] ;[508 \mathrm{C}>\mathrm{G}]$ & MHP & 10.0 & NBS & 600 & 77 & 7.79 \\
\hline 25 & c. $[60+5 \mathrm{G}>\mathrm{T}] ;[$ [754C $>\mathrm{T}]$ & cPKU & 0.0 & CD & 1206 & 33 & 36.55 \\
\hline 26 & c. $[60+5 \mathrm{G}>\mathrm{T}] ;[838 \mathrm{G}>\mathrm{A}]$ & cPKU & 0.0 & CD & 2326 & 41 & 56.73 \\
\hline
\end{tabular}

Abbreviations: cPKU, classic phenylketonuria; NBS, newborn screening; CD, clinical diagnosis; GVP, genotypic phenotype value; MHP, mild hyperphenylalaninemia; $\mathrm{mPKU}$, mild phenylketonuria; Phe, phenylalanine; Tyr, tyrosine.

A)

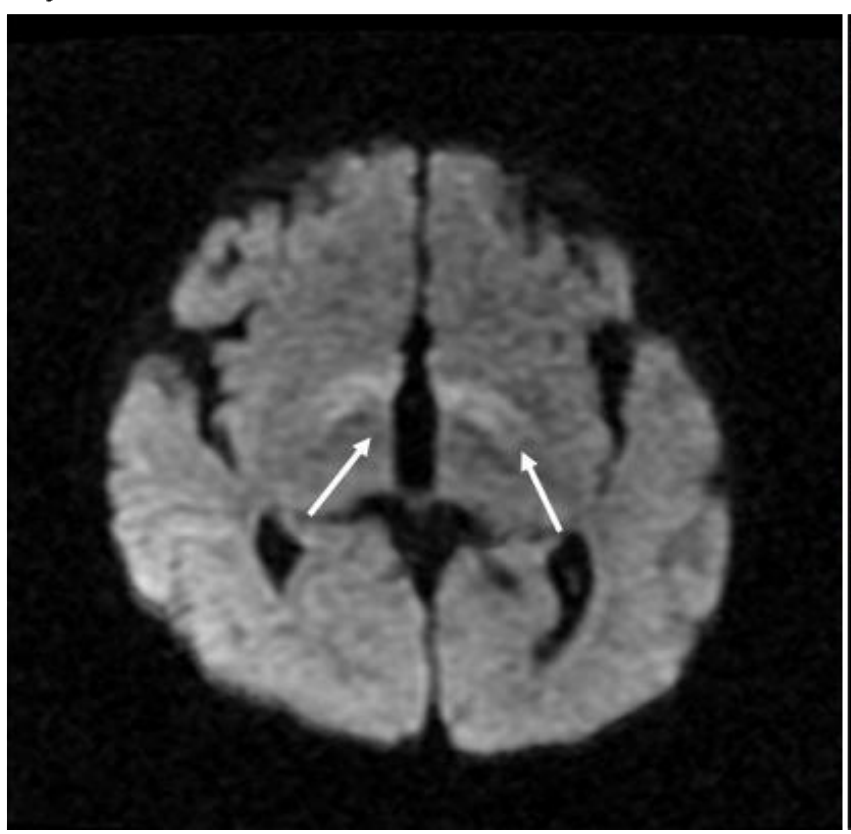

\section{B)}

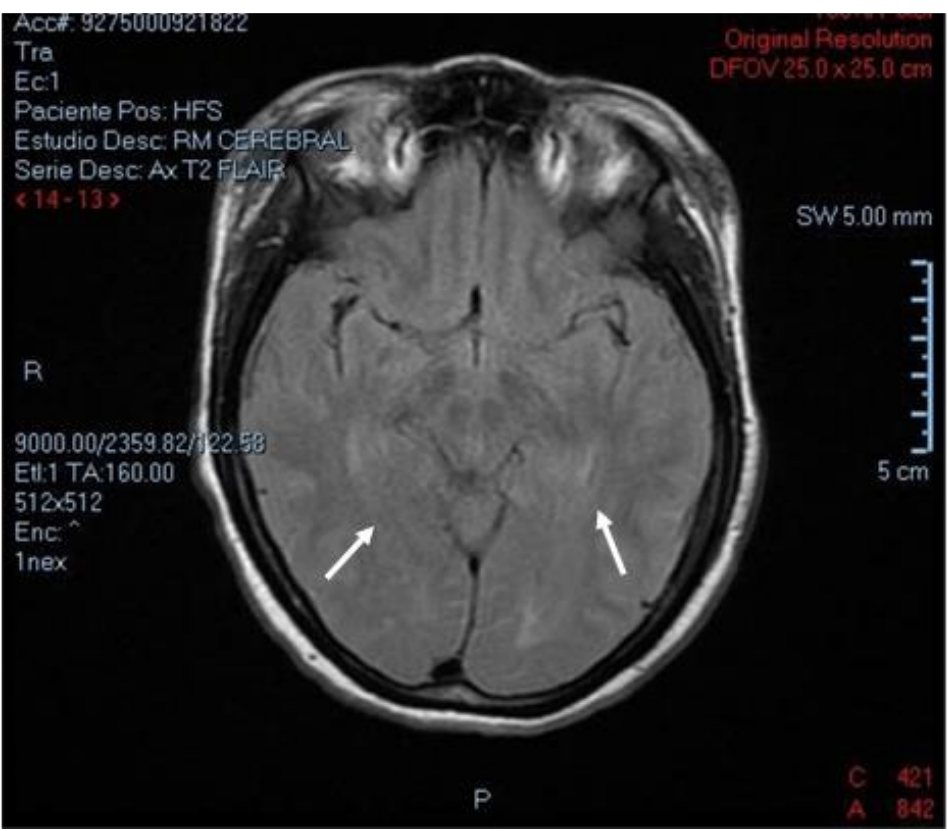

Figure 4. Brain NMRI imaging of two CD patients diagnosed with homozygous genotype c. $60+5 \mathrm{G}>\mathrm{T}$, and cPKU phenotype. (A) Diffusion NMRI of one-year-old male, showing restricted diffusion in periventricular white matter and basal ganglia (arrows). Hypomyelination and brain atrophy were also observed. (B) Axial T2 FLAIR imaging of a 10-year-old female showing periventricular white matter hyperintensity (arrows). 


\subsection{Novel PAH Variants and Description of Resulting Phenotypes}

The frameshift c. 23del or p. (Asn8Thrfs * 30) variant was found in a patient categorized as cPKU and bearing the p. (Val388Met) in the second allele (Patient 46, Table 2). Clinically, this patient showed a maximum blood Phe concentration of $2798 \mu \mathrm{mol} / \mathrm{L}$, a Tyr blood concentration of $133.29 \mu \mathrm{mol} / \mathrm{L}$, a Phe/Tyr ratio of 20.9 and a dietary Phe tolerance of $215 \mathrm{mg} /$ day. This patient began nutritional treatment at 1.5 months old, with consistent adherence to treatment during 4 years of follow up, with most of her Phe values within the therapeutical range $(<360 \mu \mathrm{mol} / \mathrm{L})$ and neurodevelopmental skills according to her age.

The c. $1315+5 \_1315+6$ insGTGTAACAG variant was identified in compound heterozygosity involving the p. (His264Arg) variant (Patient 88, Table 2) in a cPKU patient showing Phe blood levels of $1753 \mu \mathrm{mol} / \mathrm{L}$, Tyr of $75 \mu \mathrm{mol} / \mathrm{L}$, a Phe/Tyr ratio of 23.37 and dietary Phe tolerance of $255 \mathrm{mg} /$ day. The patient initiated nutritional treatment at the neonatal stage, and during a follow-up period of 5 years, he displayed normal neurodevelopmental skills according to his age. This intronic insertion is located at the $5^{\prime}$ end of intron 12 of $P A H$, and although it does not directly disturb the canonical donor splice site, the in silico evaluation agrees with the diminished recognition of the intron 12 donor splice site (MaxEntScan: $-42.9 \%$, NNSPLICE: $-8.8 \%$, SpliceSiteFinder-like: $-6.4 \%$, GeneSplicer: $-100 \%$; ESEFinder: abolition of a splicing intronic enhancer element recognized by SRp55).

The homozygous c. [625_626insC]; [625_626insC] genotype for a novel frameshift insertion was identified in a cPKU patient (Patient 72, Table 2), showing a Phe blood concentration of $1217 \mu \mathrm{mol} / \mathrm{L}$, Tyr of $58 \mu \mathrm{mol} / \mathrm{L}$, a Phe/Tyr ratio of 20.9 and a dietary Phe tolerance of $319 \mathrm{mg} /$ day. As this patient was adopted at an early age, we did not have his family history (i.e., parental consanguinity). Early instauration of dietary management at the age of 25 days allowed him to maintain most of his blood Phe values within the therapeutic range and normal neurodevelopmental skills according to his age, in a 6.5-year follow-up period.

\subsection{Protein in Silico Modeling of the p. (His264Arg) Variant}

The c. 791A > G or p. (His264Arg) has been recently reported in the BIOPKUdb, although its allele frequency is still not known, results of functional assays are not available, and its resulting biochemical phenotype is still unknown. In fact, in the ClinVar database, it is considered as a variant of unknown significance (RCV001224584.2). Herein, it was identified in two unrelated patients (ID 64 and 88, Table 2). The PolyPhen, PROVEAN, and Pmut programs unanimously predicted the $\mathrm{p}$. (His264Arg) variant as deleterious with high confidence scores. Due to the rarity of this variant, we evaluated in silico the potential disturbance at the tridimensional structural arrangement of the resulting protein. We found that the substitution of histidine by the positively charged amino acid arginine predicts a serious disturbing effect within the vicinity of the catalytic site in all the possible rotamers of arginine (Figure 5).

\subsection{Theoretical $\mathrm{BH}_{4}$ Responsiveness}

We found that there were $52 \%(65 / 124)$ of patients whose genotypes included at least one potential responder allele, or one that has still not been reported in BIOPKUdb (Table 4). The MHP was the predominant biochemical phenotype of these patients (52.3\%, $34 / 65)$, but cPKU accounted for $27.7 \%$ (18/65), and the remainder accounted for $\mathrm{mPKU}$ $(20 \%, 13 / 65)$. The most frequent responder allele was c. $1162 \mathrm{G}>\mathrm{A}$ or p. (Val388Met) (14 alleles), followed by c. 782G > A or p. (Arg261Gln) (seven alleles) and c. 722G > A or p. (Arg241His) and c. 1169A > G or p. (Gln390Gly) (six alleles each). 
Table 4. Potential $\mathrm{BH}_{4}$-responder patients $(65 / 124,52 \%)$ according to their genotype, and those who were recommended for the $\mathrm{BH}_{4}$-responsiveness test.

\begin{tabular}{|c|c|c|c|c|}
\hline \multirow[b]{2}{*}{ Genotype } & \multicolumn{4}{|c|}{$\% \mathrm{BH}_{4}$ Response Reported in BIOPKU } \\
\hline & $\begin{array}{l}\text { Observed Biochemical } \\
\text { Phenotype }\end{array}$ & $\begin{array}{l}\text { Allele } 1 \text { in Homozygous } \\
\text { State Yes/Slow }\end{array}$ & $\begin{array}{c}\text { Allele } 2 \text { in Homozygous } \\
\text { State Yes/Slow }\end{array}$ & $\mathrm{BH}_{4}$ Responsiveness \\
\hline c. $[722 \mathrm{G}>\mathrm{A}] ;[\mathrm{722 \textrm {G }}>\mathrm{A}]$ & MHP & $100 / 0$ & $100 / 0$ & \\
\hline c. $[1169 \mathrm{~A}>\mathrm{G}] ;[1169 \mathrm{~A}>\mathrm{G}]$ & MHP & $100 / 0$ & $100 / 0$ & \\
\hline c. $[1208 \mathrm{C}>\mathrm{T}] ;[1208 \mathrm{C}>\mathrm{T}]$ & MHP & $100 / 0$ & $100 / 0$ & \\
\hline c. $[722 \mathrm{G}>\mathrm{A}] ;[782 \mathrm{G}>\mathrm{A}]$ & MHP & $100 / 0$ & $74 / 4$ & \\
\hline c. $[722 \mathrm{G}>\mathrm{A}] ;[842+1 \mathrm{G}>\mathrm{A}]$ & cPKU & $100 / 0$ & 0 & \\
\hline c. $[782 \mathrm{G}>\mathrm{A}] ;[1169 \mathrm{~A}>\mathrm{G}]$ & MHP & $74 / 4$ & $100 / 0$ & Identical genotype reported \\
\hline c. $[728 \mathrm{G}>\mathrm{A}] ;[1243 \mathrm{G}>\mathrm{A}]$ & MHP & $0 / 6.67$ & NR & in BIOPKUdb as responder \\
\hline c. $[60+5 \mathrm{G}>\mathrm{T}] ;[1169 \mathrm{~A}>\mathrm{G}]$ & MHP & NR & $100 / 0$ & \\
\hline c. $[1045 \mathrm{~T}>\mathrm{C}] ;[1169 \mathrm{~A}>\mathrm{G}]$ & MHP & 0 & $100 / 0$ & \\
\hline c. $[1045 \mathrm{~T}>\mathrm{C}] ;[1208 \mathrm{C}>\mathrm{T}]$ & MHP & 0 & $100 / 0$ & \\
\hline c. $[1045 \mathrm{~T}>\mathrm{C}] ;[1243 \mathrm{G}>\mathrm{A}]$ & MHP & 0 & NR & \\
\hline c. $[60+5 \mathrm{G}>\mathrm{T}] ;[508 \mathrm{C}>\mathrm{G}]$ & MHP & NR & NR & \\
\hline c. $[204 \mathrm{~A}>\mathrm{T}] ;[527 \mathrm{G}>\mathrm{T}]$ & MHP & $100 / 0$ & NR & \multirow{46}{*}{ Potential responder } \\
\hline c. $[204 \mathrm{~A}>\mathrm{T}] ;[782 \mathrm{G}>\mathrm{A}]$ & $\mathrm{mPKU}$ & $100 / 0$ & $74 / 4$ & \\
\hline c. $[204 \mathrm{~A}>\mathrm{T}] ;[829 \mathrm{~T}>\mathrm{G}]$ & MHP & $100 / 0$ & 0 & \\
\hline c. $[721 \mathrm{C}>\mathrm{T}] ;[1066-11 \mathrm{G}>\mathrm{A}]$ & MHP & $100 / 0$ & $0 / 6.09$ & \\
\hline c. $[722 \mathrm{G}>\mathrm{A}] ;[\mathrm{728 \textrm {G } > \mathrm { A } ]}$ & MHP & $100 / 0$ & $0 / 6.67$ & \\
\hline c. $[1042 \mathrm{C}>\mathrm{G}] ;[194 \mathrm{~T}>\mathrm{C}]$ & mPKU & $100 / 0$ & $84 / 0$ & \\
\hline c. $[1042 \mathrm{C}>\mathrm{G}] ;[1238 \mathrm{G}>\mathrm{A}]$ & cPKU & $100 / 0$ & NR & \\
\hline c. $[194 \mathrm{~T}>\mathrm{C}] ;\left[208 \_210 \mathrm{del}\right]$ & cPKU & $84 / 0$ & NR & \\
\hline c. $[194 \mathrm{~T}>\mathrm{C}] ;[533 \overline{\mathrm{A}}>\mathrm{G}]$ & MHP & $84 / 0$ & NR & \\
\hline c. $[194 \mathrm{~T}>\mathrm{C}] ;[1241 \mathrm{~A}>\mathrm{G}]$ & mPKU & $84 / 0$ & $100 / 0$ & \\
\hline c. $[782 \mathrm{G}>\mathrm{A}] ;[1045 \mathrm{~T}>\mathrm{C}]$ & cPKU & $74 / 4$ & 0 & \\
\hline c. $[1162 \mathrm{G}>\mathrm{A}] ;[1162 \mathrm{G}>\mathrm{A}]$ & mPKU & $62.5 / 12.5$ & $62.5 / 12.5$ & \\
\hline c. $[1162 \mathrm{G}>\mathrm{A}] ;$ c. $[1243 \mathrm{G}>\mathrm{A}]$ & MHP & $62.5 / 12.5$ & NR & \\
\hline c. $[1162 \mathrm{G}>\mathrm{A}] ;[1315+1 \mathrm{G}>\mathrm{A}]$ & cPKU & $62.5 / 12.5$ & $0 / 13$ & \\
\hline c. $[1162 \mathrm{G}>\mathrm{A}] ;[1315+2 \mathrm{~T}>\mathrm{C}]$ & cPKU & $62.5 / 12.5$ & NR & \\
\hline c. $[898 \mathrm{G}>\mathrm{T}] ;[1162 \mathrm{G}>\mathrm{A}]$ & MHP & $60 / 40$ & $62.5 / 12.5$ & \\
\hline c. $[1055 \mathrm{del}] ;[1162 \mathrm{G}>\mathrm{A}]$ & cPKU & $5 / 5$ & $62.5 / 12.5$ & \\
\hline c. [116_118del]; [1045T > C] & cPKU & $0 / 80$ & 0 & \\
\hline c. $[117 \mathrm{C}>\mathrm{G}] ;[1157 \mathrm{~A}>\mathrm{G}]$ & mPKU & $0 / 50$ & NR & \\
\hline c. $[117 \mathrm{C}>\mathrm{G}] ;[441+5 \mathrm{G}>\mathrm{T}]$ & mPKU & $0 / 50$ & NR & \\
\hline c. $[781 \mathrm{C}>\mathrm{T}] ;[782 \mathrm{G}>\mathrm{A}]$ & cPKU & $0 / 20$ & $74 / 4$ & \\
\hline c. $[781 \mathrm{C}>\mathrm{T}] ;[1243 \mathrm{G}>\mathrm{A}]$ & MHP & $0 / 20$ & NR & \\
\hline c. $[1066-11 \mathrm{G}>\mathrm{A}] ;[1127 \mathrm{delA}]$ & mPKU & $0 / 6.09$ & NR & \\
\hline c. $[1066-11 \mathrm{G}>\mathrm{A}] ;[1162 \mathrm{G}>\mathrm{A}]$ & cPKU & $0 / 6.09$ & $62.5 / 12.5$ & \\
\hline c. $[673 \mathrm{C}>\mathrm{A}] ;[907 \mathrm{~T}>\mathrm{G}]$ & MHP & 0 & NR & \\
\hline c. $[1045 \mathrm{~T}>\mathrm{C}] ;[1162 \mathrm{G}>\mathrm{A}]$ & $\mathrm{mPKU}$ & 0 & $62.5 / 12.5$ & \\
\hline c. $[1 \mathrm{~A}>\mathrm{T}] ;[722 \mathrm{G}>\mathrm{A}]$ & MHP & NR & $100 / 0$ & \\
\hline c. $[1 \mathrm{~A}>\mathrm{T}] ;[1042 \mathrm{C}>\mathrm{G}]$ & cPKU & NR & $100 / 0$ & \\
\hline c. $[23 \mathrm{del}] ;[1162 \mathrm{G}>\mathrm{A}]$ & cPKU & NR & $62.5 / 12.5$ & \\
\hline c. $[60+5 \mathrm{G}>\mathrm{T}] ;[1162 \mathrm{G}>\mathrm{A}]$ & cPKU & NR & $62.5 / 12.5$ & \\
\hline c. [165del]; [194T > C] & cPKU & NR & $84 / 0$ & \\
\hline c. $[165 \mathrm{~T}>\mathrm{G}] ;$ [208_210del] & MHP & NR & NR & \\
\hline c. $\left[208 \_210 \mathrm{del}\right] ;[$ [28G > A] & mPKU & NR & $0 / 6.67$ & \\
\hline c. $[439 \mathrm{C}>\mathrm{T}] ;[782 \mathrm{G}>\mathrm{A}]$ & mPKU & NR & $74 / 4$ & \\
\hline c. $[441+5 \mathrm{G}>\mathrm{T}] ;[782 \mathrm{G}>\mathrm{A}]$ & cPKU & NR & $74 / 4$ & \\
\hline c. $[508 \mathrm{C}>\mathrm{G}] ;[842+1 \mathrm{G}>\mathrm{A}]$ & mPKU & NR & 0 & \\
\hline c. $[508 \mathrm{C}>\mathrm{G}] ;[1243 \mathrm{G}>\mathrm{A}]$ & MHP & NR & NR & \\
\hline c. $[508 \mathrm{C}>\mathrm{G}] ;[1315+1 \mathrm{G}>\mathrm{A}]$ & mPKU & NR & $0 / 13$ & \\
\hline c. $[533 \mathrm{~A}>\mathrm{G}] ;[1162 \mathrm{G}>\mathrm{A}]$ & MHP & NR & $62.5 / 12.5$ & \\
\hline c. $[533 \mathrm{~A}>\mathrm{G}] ;[1169 \mathrm{~A}>\mathrm{G}]$ & MHP & NR & $100 / 0$ & \\
\hline $\begin{array}{l}\text { c. }\left[791 \mathrm{~A}>\text { G]; }\left[1315+5 \_1315+\right.\right. \\
\text { 6insGTGTAACAG] }\end{array}$ & cPKU & NR & NR & \\
\hline c. $[809 \mathrm{G}>\mathrm{A}] ;[941 \mathrm{C}>\mathrm{A}]$ & MHP & NR & NR & \\
\hline c. $[809 \mathrm{G}>\mathrm{A}] ;[1162 \mathrm{G}>\mathrm{A}]$ & mPKU & NR & $62.5 / 12.5$ & \\
\hline c. $[969+6 \mathrm{~T}>\mathrm{C}] ;[1066-11 \mathrm{G}>\mathrm{A}]$ & cPKU & NR & $0 / 6.09$ & \\
\hline c. $[969+6 \mathrm{~T}>\mathrm{A}] ;[1162 \mathrm{G}>\mathrm{A}]$ & MHP & NR & $62.5 / 12.5$ & \\
\hline c. $[1243 \mathrm{G}>\mathrm{A}] ;[1315+1 \mathrm{G}>\mathrm{A}]$ & MHP & NR & $0 / 13$ & \\
\hline c. $[1 \mathrm{~A}>\mathrm{T}] ;[1243 \mathrm{G}>\mathrm{A}]$ & MHP & NR & NR & \multirow{7}{*}{ Undetermined response } \\
\hline c. $[533 \mathrm{~A}>\mathrm{G}] ;[533 \mathrm{~A}>\mathrm{G}]$ & MHP & NR & NR & \\
\hline c. $[533 \mathrm{~A}>\mathrm{G}] ;[809 \mathrm{G}>\mathrm{A}]$ & MHP & NR & NR & \\
\hline c. [625_626insC]; [625_626insC] & cPKU & NR & NR & \\
\hline c. $[688 \mathrm{G}>\mathrm{A}] ;[754 \mathrm{C}>\mathrm{T}]$ & MHP & NR & 0 & \\
\hline c. $[727 \mathrm{C}>\mathrm{T}] ;[1243 \mathrm{G}>\mathrm{A}]$ & MHP & No & NR & \\
\hline c. $[969+6 \mathrm{~T}>\mathrm{A}] ;[1198 \mathrm{~A}>\mathrm{C}]$ & MHP & NR & NR & \\
\hline
\end{tabular}

Novel pathogenic $P A H$ variants are highlighted in red bold type. Abbreviations: $c P K U$, classic phenylketonuria; MHP, mild hyperphenylalaninemia; $\mathrm{mPKU}$, mild phenylketonuria; NR, not reported or still undetermined. 
A)



B)
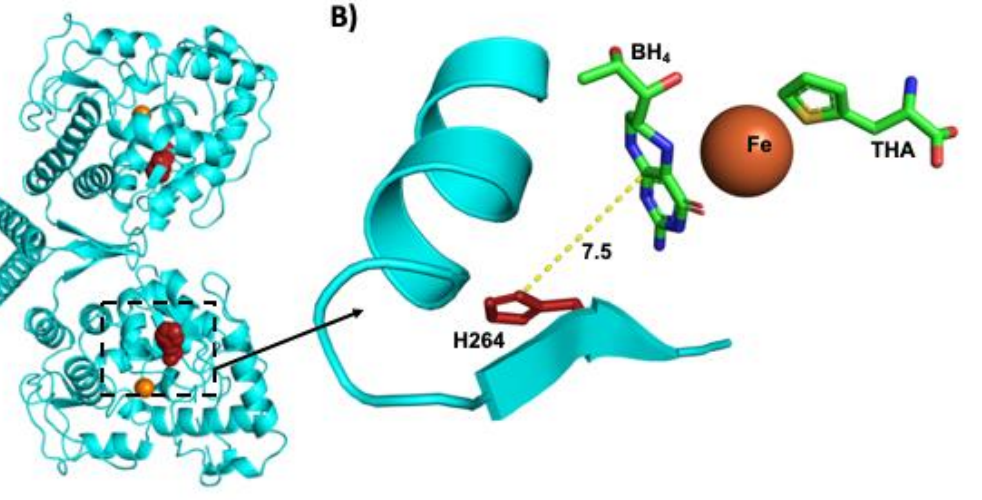

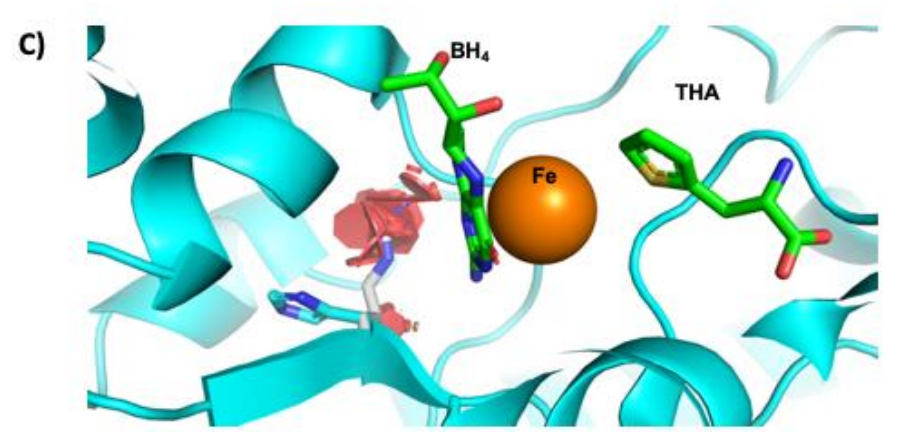

Figure 5. Location of His-264 residue in PAH structure. (A) His-264 residue is located within the vicinity of the active site; (B) zoom of His-264 location that shows a distance of $7.5 \AA$ from the catalytic PAH site; (C) illustration of resulting repulsive clashes (red hexagons) conditioned by the His residue substitution by the positively charged Arg residue. The figure was constructed with Pymol [13]. THA = 3-(2-thienyl)-L-alanine (phenylalanine analogue).

\section{Discussion}

The epidemiology of PKU has been the objective of a growing number of publications $[2,3,18]$; however, the main findings of those investigations are based on European and Asiatic populations. The Latin American population is poorly represented, mainly due to the scarce reliable published data from this region. In a recent PKU work, genetic and epidemiological data from 64 countries were presented [2]; however, only three Latin American countries were considered (Argentina, Brazil and Mexico $[6,19,20])$, so there would be a possible underrepresentation of the mutational $P A H$ spectrum in Latin American HPA/PKU patients. The present study aimed to update the PAH mutational spectrum of Mexican HPA/PKU patients attending a single reference center. Moreover, to the best of our knowledge, this is the largest published cohort of PKU patients from Mexico, including individuals of almost all the geographic regions of the country (patients came from $30 / 32$ of the states that constitute the country).

In this new study, patients' phenotypes were classified according to the criteria established by Hillert et al. and van Spronsen et al. [2,3], which will facilitate international comparisons both with BIOPKUdb and other publications. By comparing the frequencies of the different $P A H$-related biochemical phenotypes identified herein with those previously reported [6], no statistical differences were found.

The number of patients detected by NBS in our center increased from $54.1 \%$ in 2015 [6] to $78.2 \%$. This significant increment $(p=0.001)$ could be related to the optimization of the NBS system in Mexico, although it still does not reach the optimal goal of having a total NBS coverage for this disease [21]. This finding agrees with the fact that improvements in the NBS programs increase the detection of MHP forms [22,23]. Even though it is widely accepted that MHP patients are only treated when Phe blood levels are $>360 \mu \mathrm{mol} / \mathrm{L}$, the biochemical follow up of these patients should be periodical, in order to assure neuro- 
logically safe Phe values [24]. Moreover, in female patients at a reproductive stage, a close follow up is especially relevant considering the potential risk of their offspring suffering from maternal PKU syndrome [2].

As reported has been worldwide [2,3], the molecular PKU spectrum found in the present study is heterogeneous, and variants are distributed along the entire gene with clustering in exons that codify the catalytic PAH region (35\%), especially exons 7 and 6 (Figure 3). Notably, we did not find any patient with the "Celtic" c. 1222C > T or p. (Arg408Trp) pathogenic variant, which is considered the most frequent one worldwide, especially in central and eastern Europe (44.4-53.7\%); however, its allele frequency in the Spanish population decreased significatively ( $4 \%$ ), which could correlate with its absence in the Mexican population, which is characterized by bearing a Spanish-derived ancestry [3]. On the other hand, the absence of variants in exon 13 (Figure 3) was similar to that observed in Spain and other Latin American populations [19,25-28]. This fact is in agreement with the low number of pathogenic variants registered in exon 13 in BIOPKUdb.

The Sanger methodology allowed us to obtain a detection rate of $87.3 \%(124 / 142)$ of the biallelic PAH genotypes in the patients initially classified as HPA/PKU; however, this methodology identified that there were $2.81 \%$ (4/142) of patients carrying only one pathogenic allele (Figure 1). To date, there are no available data to estimate the proportion of large rearrangements unnoticed for Sanger sequencing methodology, which are expected in less than 1\% of $P A H$-related HPA patients worldwide [3]. At least in Russian patients, gross exonic deletions (mainly involving exon 3 and 5) identified by Multiplex Ligationdependent Probe Amplification (MLPA) analysis account for $0.39 \%$ of pathogenic PAH alleles [29]. Thus, in these four patients with monoallelic genotypes-who presented persistent HPA, three of which required nutritional treatment-further studies, such as MLPA analysis, could be an option in order to discard gross $P A H$ rearrangements.

The remaining 14 cases (9.8\%) with normal $P A H$ genotypes lacked the recommended former dihydropteridine reductase (DHPR) and pterin evaluations [2] to discard a defect in the metabolism of $\mathrm{BH}_{4}$.

Our study corroborates the high frequency of the c. $60+5 \mathrm{G}>\mathrm{T}$ variant $(14.5 \%$, Table 1$)$ and its geographical predominance in the western and central regions of Mexico (Table 2), probably due to the founder effect [6]; this has been corroborated by other authors [9]. This intronic null variant, formerly known as "IVS1 $+5 \mathrm{~g}>\mathrm{t}$ ", was first described by Guldberg et al. in 1993 [30]. It is a rare variant reported in only $0.7 \%$ and $1.36 \%$ of Danish [30] and Spanish [31] populations, respectively. In BIOPKUdb, it is reported in $0.32 \%$ of the subjects. Due to its rarity, it has been scarcely studied. Interestingly, this variant has not been found in PKU patients from Argentina, Chile or Cuba [19,27,32], but it has been reported in Costa Rica [33], and Brazil [20,25,34,35]. Moreover, the c. $60+5 \mathrm{G}>\mathrm{T}$ variant has not been reported in Russia [36], Japan [23], or China [37]. Therefore, to the best of our knowledge, the studied Mexican population has the highest frequency of this variant.

In the present study, all the patients harboring a homozygous c. $60+5 \mathrm{G}>\mathrm{T}$ genotype had cPKU, which can be explained by the severity of this null variant (APV value $=0$ ), which can theoretically lead to no enzymatic activity, in addition to an absence of $\mathrm{BH}_{4}$ response [15]. As expected, among patients harboring c. $60+5 \mathrm{G}>\mathrm{T}$ in compound heterozygosity involving a less severe allele with APV $>5$ [14], the disease was less severe, corresponding to an MHP phenotype (patients 23 and 24, Table 3), confirming that functionally mild variants with a substantial residual PAH enzymatic activity dominate over null alleles [3]. The clinical picture of patients with homozygous genotypes c. [60 + 5G > T]; $[60+5 \mathrm{G}>\mathrm{T}]$ coincides with that described for cPKU patients, consisting of early, severe and progressive neurological manifestations, and symptomatology worsening if the start of dietary treatment is delayed. Therefore, these findings emphasize the importance of starting treatment in the first days of life, preferably in the first week [8], to prevent brain damage that leads to neurodevelopmental delay and permanent intellectual disability [38]. At least in two c. $60+5 \mathrm{G}>$ T-homozygous and lately diagnosed patients (CD group), we documented central nervous system sequelae (Figure 4), consisting of basal ganglia 
and white matter brain damage. Remarkably, although the male patient was diagnosed at a younger age, he showed the involvement of basal ganglia, but it is known that imaging studies of PKU patients do not always correlate with the severity of the observed phenotype [39].

The second most frequent variant of this work was c. $1162 \mathrm{G}>\mathrm{A}$ p. (Val388Met) $(11.2 \%)$. This variant is common in the Spanish population $(6.8 \%)$ [26], as well as in other Latin American countries such as Chile (17.2\%) [27], Argentina (3.9\%) [19] and Brazil $(12.7-21.2 \%)$ [25,28]. Interestingly, our five homozygous p. (Val388Met) patients had different biochemical phenotypes (mPKU: $N=4$ and cPKU: $N=1$, Table 2). The predominance of mPKU phenotypes observed herein associated with homozygous p. (Val388Met) contrasts with that reported in BIOPKUdb, where half of the patients show cPKU. Furthermore, this variant was the most frequently found in the discordant heterozygous genotypes (4/12) and in the potential $\mathrm{BH}_{4}$-responder genotypes (13/60, Table 4). Its predominance among patients with an inconsistent phenotype could be related to diverse proposed phenomena, such as its destabilizing PAH nature; differences in protein folding; or other, less known factors, such as the influence of modifier genes or epigenetic modifications [26].

The c. $441+5 \mathrm{G}>\mathrm{T}$ or p. (?) intronic and severe variant $(\mathrm{APV}=0)$, was found in $5.64 \%$ of the studied alleles, which contrasts with its low frequency worldwide (0.98\%). However, higher allelic frequencies have been reported for other Latin American countries, such as Argentina (2.4\%) [19] and Chile (3.0\%) [27]. Severe cPKU is expected for those homozygous individuals bearing this null allele. Remarkably, in the present study, 50\% of the patients with this variant, including two homozygous patients, are originally from the north of Mexico (Baja California, Chihuahua, Coahuila and Tamaulipas). This likely suggests a possible founder effect similar to that described for c. $60+5 G>T$ [6]; therefore, further research is needed to support this hypothesis.

The c. 1066-11G > A or p. (Gln355_Tyr356insGlyLeuGln) variant has an APV =0 leading to the cPKU phenotype. It was identified in $5.6 \%$ of the alleles (Table 1 ), which is similar to that reported in BIOPKUdb (6.8\%), Argentina (9.3\%), Brazil (9.4\%) [3] and Chile $(12.7 \%)$ [27]. This variant was previously proposed as being responsible for the high PKU cases from Jalisco State [40]; however, our work does not support this previous asseveration, since we did not observe any geographical predominance for patients carrying this variant.

Regarding the novel c. 1315 + 5_1315 + 6insGTGTAACAG variant, it is currently classified as likely pathogenic, as it meets the PM2, PM3, PP3 and PP4 ACMG/AMP criteria [11]. The paternal origin of this variant was confirmed in cPKU Patient \#88, who has an extremely low dietary Phe tolerance ( $255 \mathrm{mg} /$ day) associated with his compound heterozygous genotype involving c. 791A > G or p. (His264Arg) of maternal origin (Table 2). This last missense variant was recently posted in BIOPKUdb, and we predicted it to be a pathogenic change (Figure 5), supported by PM1, PM2, PM5, PP3 and PP4 ACMG/AMP criteria [11].

The novel c. 625_626insC variant was found in homozygous state in patient \#77 affected by cPKU (Table 2), in addition to low dietary Phe tolerance ( $319 \mathrm{mg} /$ day), but no familial antecedents were available as the patient was adopted. This frameshift variant was classified as pathogenic according to the ACMG/AMP (American College of Medical Genetics / Association for Molecular Pathology) criteria (very strong pathogenic variant (PVS1), pathogenic moderate (PM1, PM2) and PP4 ACMG/AMP) [11].

The third novel c. 23del or p. (Asn8Thrfs * 30) frameshift variant was also classified as pathogenic, as it meets the PVS1, PM1, PM2 and PP4 ACMG/AMP criteria [11]. This variant was in heterozygous state with c. 1162G > A or p. (Val388Met) for patient \#46 (Table 2), who was classified as a cPKU with low Phe dietary tolerance ( $215 \mathrm{mg} /$ day). To our knowledge, this variant has never been documented; however, Wang et al., 2018, reported a similar c. 23_24delinsT or p. (Asn8Ilefs * 30) variant (PAH1043 accession number BIOPKUdb) [41]. Although it is a different variant, the resulting frameshift effect at the regulatory domain is the same. The occurrence of these two indels at the same position could suggest a "hot spot" for small rearrangements in c. 23 and c. 24 positions. 
In the present work, the observed general genotype/phenotype concordance was $83.8 \%$ when compared with identical genotypes reported in the BIOPKUdb. These data are slightly higher than previously reported by our group $(70.8 \%)$ [6]. This higher concordance is likely due to the new classification criteria used, although other unknown epigenetic or environmental regulatory factors could be involved. One limitation for the frequency of variants and concordance comparison is that not all patients from different populations are listed in the BIOPKUdb there are few identical genotypes, especially those with rare variants, hence the importance of contributing to this database with the information of the $P A H$ mutational spectrum from diverse world regions. Interestingly, in this study, discordance consisted mainly of the underestimation of the disease severity $(8 / 12,66.7 \%)$, as we classified patients with a less severe disease type. This could highlight the difficulties of establishing the correct phenotype based only on blood Phe levels and the importance of establishing the genotype in all HPA/PKU patients [42].

Finally, we found that $52 \%$ of the patients were considered potential $\mathrm{BH}_{4}$ responders (Table 4). This is similar to the worldwide responsiveness reported by Hillert et al., who found $43 \%$ responsiveness from a cohort of 5597 subjects [3]. The most frequent potential $\mathrm{BH}_{4}$ responder variants are of the missense type, with residual enzymatic activity that could be enhanced with pharmacological doses of $\mathrm{BH}_{4}$ [43]. On the other hand, we emphasize that patients carrying two non-responder alleles in trans should not be submitted to the $\mathrm{BH}_{4}$ response test, since those variants $(27 / 60,45 \%)$ do not allow the synthesis of a functional protein; thus, the recommendation for those patients is to maintain a strictly nutritional treatment $[15,16]$.

\section{Conclusions}

In summary, the high number of variants found, including the three new variants, confirms the heterogeneous and complex mutational landscape of PKU in Mexico. Half of the studied Mexican patients had cPKU, with a predominance of the c. $60+5 \mathrm{G}>\mathrm{T}$ variant (14.5\% of the alleles), which could indicate the highest allelic frequency reported for this variant worldwide. Furthermore, we highlight the severe central nervous system sequelae in two late-diagnosed symptomatic c. $60+5 \mathrm{G}>$ T-homozygous patients which points out the importance of an early instauration of dietary treatment. A high genotype-phenotype concordance was observed ( $>80 \%)$. A possible founder effect of the c. $441+5 \mathrm{G}>\mathrm{T}$ variant in the north of Mexico warrants further research.

Supplementary Materials: The following are available online at https:/ /www.mdpi.com/article/10 .3390/genes12111676/s1, Table S1: PCR oligonucleotides and sequencing conditions for PAH gene.

Author Contributions: Conceptualization, M.V.-A., M.A.A.-O., I.I.-G. and C.F.-L.; Data curation, M.A.A.-O., I.I.-G., A.G.-d.A., L.F.-H. and C.F.-L.; Formal analysis, M.V.-A., M.A.A.-O., A.G.-d.A. and L.F.-H.; Funding acquisition, M.V.-A., M.A.A.-O., A.G.-d.A. and L.F.-H.; Investigation, M.V.-A., M.A.A.-O., A.G.-d.A., L.F.-H., S.G.-L., L.L.-M., R.I.C.-N. and L.B.-M.; Methodology, M.A.A.-O., A.G.-d.A., S.G.-L., L.L.-M. and R.I.C.-N.; Project administration, M.V.-A., M.A.A.-O. and C.F.-L.; Resources, M.V.-A., M.A.A.-O., I.I.-G. and A.G.-d.A.; Software, M.A.A.-O., I.I.-G., L.F.-H. and C.F.-L.; Supervision, M.A.A.-O. and C.F.-L.; Validation, M.A.A.-O., I.I.-G., L.F.-H. and C.F.-L.; Visualization, M.A.A.-O., A.G.-d.A., L.F.-H. and C.F.-L.; Writing-original draft, M.V.-A., M.A.A.-O., I.I.-G. and C.F.-L.; Writing-review and editing, M.V.-A., M.A.A.-O., I.I.-G., A.G.-d.A., L.F.-H., S.G.-L., L.L.-M., R.I.C.-N., L.B.-M. and C.F.-L. All authors have read and agreed to the published version of the manuscript.

Funding: This research was funded by the National Institute of Pediatrics (Recursos Fiscales 2018-2020, Programa E022 Investigación y Desarrollo Tecnológico en Salud, Ciudad de México, Mexico).

Institutional Review Board Statement: This study was approved prior to data collection by the research, biosecurity and ethics committees of the National Institute of Pediatrics (approval numbers 2010/30 and 2020/014). 
Informed Consent Statement: All participants provided written consent of their participation and the publication of data in an anonymized form.

Data Availability Statement: The datasets analyzed during the present study are available from the corresponding author on reasonable request.

Acknowledgments: We thank the patients, their families, and the Asociación Mexicana de Fenilcetonuria, A.C. (Laura Patricia Camacho Chavarín and José de Jesús Muñoz Navarro) for their support and commitment. The authors gratefully acknowledge Chemist Aída Jannet Hernández Montiel, Luis Ricardo Morales González and Jaime Torres Marcial for their technical assistance.

Conflicts of Interest: The authors declare no conflict of interest. The funders had no role in the design of the study; in the collection, analyses, or interpretation of data; in the writing of the manuscript, or in the decision to publish the results.

\section{Appendix A}

Overview of PCR and Sanger Sequencing Conditions: All reactions were carried out in $30 \mu \mathrm{L}$ under the standard conditions recommended for HotStarTaq ${ }^{\circledR}$ DNA Polymerase (www.qiagen.com/HB-0452, QIAGEN GmbH, Hilden, Germany, accessed on 10 October 2019), with 5-15 ng of genomic DNA per reaction and $0.1 \mu \mathrm{M}$ of each primer. PCR primers to cover the entire coding exon and its exon-intron or untranslated regions borders were designed with the Primer BLAST designing tool (https: / / www.ncbi.nlm.nih.gov/tools / primer-blast/, accessed on 10 October 2019) using the NG_008690.2 PAH RefSeqGene. The utilized annealing temperature was $58^{\circ} \mathrm{C}$ (exon 12) or $64^{\circ} \mathrm{C}$ (rest of exons). All reactions were carried out with a final concentration of 0.3M of betaine (MP Biomedicals, LLC., Fountain Parkway Solon, OH, USA). All PCR products were evaluated by agarose gel electrophoresis, subjected to further enzymatic purification (ExoSAP-IT ${ }^{\circledR}$ PCR Product Cleanup, Affymetrix, Inc., Santa Clara, CA, USA), and then unidirectionally sequenced with the universal M13F primer (5'-GTAAAACGACGGCCAGT-3') and Big Dye ${ }^{\circledR}$ Terminator Cycle Sequencing chemistry (Life Technologies Corp.; performed at PSOMAGEN Inc., Rockville, MD, USA). Clinically relevant variants were confirmed by performing oppositestrand sequencing with the attached universal primer. Variant annotation compliant with Human Genome Variation Society nomenclature (https: / /varnomen.hgvs.org/, accessed on 20 December 2020) was performed using the Alamut ${ }^{\circledR}$ Visual version 2.15 software (SOPHiA GENETICS, Lausanne, Switzerland).

\section{References}

1. Blau, N.; van Spronsen, F.J.; Levy, H.L. Phenylketonuria. Lancet 2010, 376, 1417-1427. [CrossRef]

2. Van Spronsen, F.J.; Blau, N.; Harding, C.; Burlina, A.; Longo, N.; Bosch, A.M. Phenylketonuria. Nat. Rev. Dis. Primers 2021, 7, 1-19. [CrossRef]

3. Hillert, A.; Anikster, Y.; Belanger-Quintana, A.; Burlina, A.; Burton, B.K.; Carducci, C.; Chiesa, A.E.; Christodoulou, J.; Đorđević, M.; Desviat, L.R.; et al. The genetic landscape and epidemiology of phenylketonuria. Am. J. Hum. Genet. 2020, 107, 234-250. [CrossRef] [PubMed]

4. Amieva, M.V.; González, I.I.; Pérez, L.D.A.H.; Caamal-Parra, G.; Martínez, L.B.; Flores, E.P.G. Epidemiología de la fenilcetonuria obtenida mediante tamiz neonatal. Acta Pediátrica Méxic 2018, 39, 25-34S. [CrossRef]

5. Bortoluzzi, V.T.; Dutra Filho, C.S.; Wannmacher, C.M.D. Oxidative stress in phenylketonuria-Evidence from human studies and animal models, and possible implications for redox signaling. Metab. Brain Dis. 2021, 36, 1-21. [CrossRef] [PubMed]

6. Vela-Amieva, M.; Abreu-Gonzalez, M.; Gonzalez-del Angel, A.; Ibarra-Gonzalez, I.; Fernandez-Lainez, C.; Barrientos-Rios, R.; Monroy-Santoyo, S.; Guillén-López, S.; Alcántara-Ortigoza, M. Phenylalanine hydroxylase deficiency in Mexico: Genotypephenotype correlations, BH4 responsiveness and evidence of a founder effect. Clin. Genet. 2015, 88, 62-67. [CrossRef] [PubMed]

7. Camp, K.M.; Parisi, M.A.; Acosta, P.B.; Berry, G.T.; Bilder, D.A.; Blau, N.; Bodamer, O.A.; Brosco, J.P.; Brown, C.S.; Burlina, A.B.; et al. Phenylketonuria scientific review conference: State of the science and future research needs. Mol. Genet. Metab. 2014, 112, 87-122. [CrossRef] [PubMed]

8. Van Wegberg, A.M.J.; Macdonald, A.; Ahring, K.; BãLanger-Quintana, A.; Blau, N.; Bosch, A.M.; Burlina, A.; Campistol, J.; Feillet, F.; Giżewska, M.; et al. The complete European guidelines on phenylketonuria: Diagnosis and treatment. Orphanet J. Rare Dis. 2017, 12, 1-56. [CrossRef] [PubMed] 
9. Russo-Estavillo, C.; Vázquez-Avelar, S.; García-Ortíz, J.E.; Real-Guerrero, J.; Belmont-Martínez, L.; Escoto-Delgadillo, M.; Hernández-Orozco, A.A.; Torres-Mendoza, B.M. High frequency of severe phenylketonuria in Jalisco, Mexico. Int. J. Hum. Genet. 2018, 18, 1-6.

10. Qureshi, G.A.; Fohlin, L.; Bergström, J. Application of high-performance liquid chromatography to the determination of free amino acids in physiological fluids. J. Chromatogr. A 1984, 297, 91-100. [CrossRef]

11. Richards, S.; Aziz, N.; Bale, S.; Bick, D.; Das, S.; Gastier-Foster, J.; Grody, W.W.; Hegde, M.; Lyon, E.; Spector, E.; et al. Standards and guidelines for the interpretation of sequence variants: A joint consensus recommendation of the American College of Medical Genetics and Genomics and the Association for Molecular Pathology. Genet. Med. 2015, 17, 405-423. [CrossRef] [PubMed]

12. DeLano, W.L. Pymol: An open-source molecular graphics tool. CCP4 Newsl. Protein Crystallogr. 2002, 40, 82-92.

13. Andersen, O.A.; Flatmark, T.; Hough, E. Crystal structure of the ternary complex of the catalytic domain of human phenylalanine hydroxylase with tetrahydrobiopterin and 3-(2-Thienyl)-l-alanine, and its implications for the mechanism of catalysis and substrate activation. J. Mol. Biol. 2002, 320, 1095-1108. [CrossRef]

14. Garbade, S.F.; Shen, N.; Himmelreich, N.; Haas, R.; Trefz, F.K.; Hoffmann, G.F.; Burgard, P.; Blau, N. Allelic phenotype values: A model for genotype-based phenotype prediction in phenylketonuria. Genet. Med. 2018, 21, 580-590. [CrossRef]

15. Van Spronsen, F.J.; van Wegberg, A.M.; Ahring, K.; Bélanger-Quintana, A.; Blau, N.; Bosch, A.M.; Burlina, A.; Campistol, J.; Feillet, F.; Giżewska, M.; et al. Key European guidelines for the diagnosis and management of patients with phenylketonuria. Lancet Diabetes Endocrinol. 2017, 5, 743-756. [CrossRef]

16. Muntau, A.C.; Adams, D.J.; Bélanger-Quintana, A.; Bushueva, T.V.; Cerone, R.; Chien, Y.-H.; Chiesa, A.; Coşkun, T.; Heras, J.D.L.; Feillet, F.; et al. International best practice for the evaluation of responsiveness to sapropterin dihydrochloride in patients with phenylketonuria. Mol. Genet. Metab. 2019, 127, 1-11. [CrossRef]

17. Buratti, E.; Chivers, M.; Královičová, J.; Romano, M.; Baralle, M.; Krainer, A.; Vořechovský, I. Aberrant 5’ splice sites in human disease genes: Mutation pattern, nucleotide structure and comparison of computational tools that predict their utilization. Nucleic Acids Res. 2007, 35, 4250-4263. [CrossRef]

18. Foreman, P.K.; Margulis, A.V.; Alexander, K.; Shediac, R.; Calingaert, B.; Harding, A.; Pladevall-Vila, M.; Landis, S. Birth prevalence of phenylalanine hydroxylase deficiency: A systematic literature review and meta-analysis. Orphanet J. Rare Dis. 2021, 16, 1-18. [CrossRef] [PubMed]

19. Enacán, R.E.; Miñana, M.N.; Fernandez, L.; Valle, M.G.; Salerno, M.; Fraga, C.I.; Santos-Simarro, F.; Prieto, L.; Lapunzina, P.; Specola, N.; et al. Phenylalanine hydroxylase (PAH) genotyping in PKU argentine patients. J. Inborn Errors Metab. Screen. 2019, 7. [CrossRef]

20. Acosta, A.; Silva Jr, W.; Carvalho, T.; Gomes, M.; Zago, M. Mutations of the phenylalanine hydroxylase (PAH) gene in Brazilian patients with phenylketonuria. Hum. Mutat. 2001, 17, 122-130. [CrossRef]

21. García-Flores, E.P.; Herrera-Maldonado, N.; Hinojosa-Trejo, M.A.; Vergara-Vázquez, M.; Halley-Castillo, M.E. Avances y logros del programa de tamiz metabólico neonatal (2012-2018). Acta Pediátrica México 2018, 39, 57-65S. [CrossRef]

22. Ohlsson, A.; Bruhn, H.; Nordenström, A.; Zetterström, R.H.; Wedell, A.; von Döbeln, U. The spectrum of PAH mutations and increase of milder forms of phenylketonuria in sweden during 1965-2014. In JIMD Reports; Springer: Berlin/Heidelberg, Germany, 2016; Volume 34, pp. 19-26. [CrossRef]

23. Odagiri, S.; Kabata, D.; Tomita, S.; Kudo, S.; Sakaguchi, T.; Nakano, N.; Yamamoto, K.; Shintaku, H.; Hamazaki, T. Clinical and genetic characteristics of patients with mild hyperphenylalaninemia identified by newborn screening program in Japan. Int. J. Neonatal Screen. 2021, 7, 17. [CrossRef]

24. Viall, S.; Ayyub, O.; Rasberry, M.; Lyons, K.; Mew, N.A. “Mild” hyperphenylalaninemia? A case series of seven treated patients following newborn screening. Mol. Genet. Metab. 2017, 122, 153-155. [CrossRef]

25. Neto, E.V.; Laranjeira, F.; Quelhas, D.; Ribeiro, I.; Seabra, A.; Mineiro, N.; Carvalho, L.D.M.; Lacerda, L.; Ribeiro, M.G. Mutation analysis of the PAH gene in phenylketonuria patients from Rio de Janeiro, Southeast Brazil. Mol. Genet. Genom. Med. 2018, 6, 575-591. [CrossRef]

26. Aldámiz-Echevarría, L.; Llarena, M.; Bueno, M.A.; Dalmau, J.; Vitoria, I.; Fernández-Marmiesse, A.; Andrade, F.; Blasco, J.; Alcalde, C.; Gil, D. Molecular epidemiology, genotype-phenotype correlation and BH 4 responsiveness in Spanish patients with phenylketonuria. J. Hum. Genet. 2016, 61, 731-744. [CrossRef]

27. Hamilton, V.; María, L.S.; Fuenzalida, K.; Morales, P.; Desviat, L.R.; Ugarte, M.; Pérez, B.; Cabello, J.F.; Cornejo, V. Characterization of phenyalanine hydroxylase gene mutations in chilean PKU patients. In JIMD Reports; Springer: Berlin/Heidelberg, Germany, 2017; Volume 42, pp. 71-77. [CrossRef]

28. Santos, L.; Castro-Magalhaes, M.; Fonseca, C.; Starling, A.; Januário, J.; Aguiar, M.; Carvalho, M. PKU in minas Gerais state, Brazil: Mutation analysis. Ann. Hum. Genet. 2008, 72, 774-779. [CrossRef]

29. Kuznetcova, I.; Gundorova, P.; Ryzhkova, O.; Polyakov, A. The study of the full spectrum of variants leading to hyperphenylalaninemia have revealed 10 new variants in the PAH gene. Metab. Brain Dis. 2019, 34, 1547-1555. [CrossRef]

30. Guldberg, P.; Henriksen, K.F.; Güttler, F. Molecular analysis of phenylketonuria in Denmark: $99 \%$ of the mutations detected by denaturing gradient gel electrophoresis. Genomics 1993, 17, 141-146. [CrossRef] [PubMed]

31. Bueno, M.A.; González-Lamuño, D.; Delgado-Pecellín, C.; Aldámiz-Echevarría, L.; Pérez, B.; Desviat, L.R.; Couce, M.L. Molecular epidemiology and genotype-phenotype correlation in phenylketonuria patients from South Spain. J. Hum. Genet. 2013, 58, 279-284. [CrossRef] 
32. Gutiérrez-García, E.; Barrios-García, B.; Gutiérrez-Gutiérrez, R.; Damiani-Rossel, A. Caracterización molecular de fenilcetonúricos cubanos. Revista Cubana de Pediatría. Rev. Cuba. Pediatría 2002, 74, 101-105.

33. Santos, M.; Kuzmin, A.I.; Eisensmith, R.C.; Goltsov, A.A.; Woo, S.L.; Barrantes, R.; De Céspedes, C. Phenylketonuria in costa rica: Preliminary spectrum of PAH mutations and their associations with highly polymorphic haplotypes. Hum. Hered. 1996, 46, 128-131. [CrossRef]

34. Vieira Neto, E.; Laranjeira, F.; Quelhas, D.; Ribeiro, I.; Seabra, A.; Mineiro, N.; Carvalho, L.M.; Lacerda, L.; Ribeiro, M.G. Genotype-phenotype correlations and BH4 estimated responsiveness in patients with phenylketonuria from Rio de Janeiro, Southeast Brazil. Mol. Genet. Genom. Med. 2019, 7, e610. [CrossRef]

35. Tresbach, R.H.; Sperb-Ludwig, F.; Ligabue-Braun, R.; Tonon, T.; Cardoso, M.T.D.O.; Heredia, R.S.; Rosa, M.T.A.D.S.; Martins, B.C.; Poubel, M.O.; Da Silva, L.C.S.; et al. Phenylketonuria diagnosis by massive parallel sequencing and genotype-phenotype association in Brazilian patients. Genes 2020, 12, 20. [CrossRef]

36. Gundorova, P.; Stepanova, A.A.; Kuznetsova, I.A.; Kutsev, S.I.; Polyakov, A.V. Genotypes of 2579 patients with phenylketonuria reveal a high rate of BH4 non-responders in Russia. PLOS ONE 2019, 14, e0211048. [CrossRef] [PubMed]

37. Zhang, X.; Ye, J.; Shen, N.; Tao, Y.; Han, L.; Qiu, W.; Zhang, H.; Liang, L.; Fan, Y.; Wang, J.; et al. In vitro residual activities in 20 variants of phenylalanine hydroxylase and genotype-phenotype correlation in phenylketonuria patients. Gene 2019, 707, 239-245. [CrossRef] [PubMed]

38. Bsc, R.A.F.E.; Wegberg, A.M.J.; Anjema, K.; Lubout, C.M.A.; Dam, E.; van Vliet, D.; Blau, N.; Spronsen, F.J. The first European guidelines on phenylketonuria: Usefulness and implications for $\mathrm{BH} 4$ responsiveness testing. J. Inherit. Metab. Dis. 2019, 43, 244-250. [CrossRef]

39. Jiménez-Pérez, M.O.; Gómez-Garza, G.; Fernández-Lainez, C.; Ibarra-González, I.; Vela-Amieva, M.; Ruiz-García, M. Resonancia magnética nuclear de encéfalo en pacientes con fenilcetonuria diagnosticada tardíamente. Acta Pediátrica México 2015, 36, 9. [CrossRef]

40. Velázquez, A.; Bilbao, G.; González-Trujillo, J.L.; Hernández, D.; Pérez-Andrade, M.E.; Vela, M.; Cicerón, I.; Loera-Luna, A.; Cederbaum, S.; Phoenix, B. Apparent higher frequency of phenylketonuria in the Mexican state of Jalisco. Hum. Genet. 1996, 97, 99-102. [CrossRef]

41. Wang, R.; Shen, N.; Ye, J.; Han, L.; Qiu, W.; Zhang, H.; Liang, L.; Sun, Y.; Fan, Y.; Wang, L.; et al. Mutation spectrum of hyperphenylalaninemia candidate genes and the genotype-phenotype correlation in the Chinese population. Clin. Chim. Acta 2018, 481, 132-138. [CrossRef]

42. Quirk, M.E.; Dobrowolski, S.F.; Nelson, B.E.; Coffee, B.; Singh, R.H. Utility of phenylalanine hydroxylase genotype for tetrahydrobiopterin responsiveness classification in patients with phenylketonuria. Mol. Genet. Metab. 2012, 107, 31-36. [CrossRef]

43. Muntau, A.C.; Röschinger, W.; Habich, M.; Demmelmair, H.; Hoffmann, B.; Sommerhoff, C.P.; Roscher, A.A. Tetrahydrobiopterin as an alternative treatment for mild phenylketonuria. N. Engl. J. Med. 2002, 347, 2122-2132. [CrossRef] [PubMed] 